\title{
The Spatial Distribution and Kinematics of Stellar Populations in E+A Galaxies
}

\author{
Stuart A. Norton ${ }^{1}$, Karl Gebhardt ${ }^{1,2}$, Ann I. Zabludoff ${ }^{3}$ and Dennis Zaritsky ${ }^{3}$
}

\begin{abstract}
We use long-slit spectroscopic observations of the sample of $\mathrm{E}+\mathrm{A}$ galaxies described by Zabludoff et al. to constrain the nature of the progenitors and remnants of the $\mathrm{E}+\mathrm{A}$ phase of galaxy evolution. We measure spatially-resolved kinematic properties of the young $(\lesssim 1$ Gyr) and old ( $\gtrsim$ few Gyr) stellar populations. The young stellar populations are more centrally concentrated than the older populations, but they are not confined to the galaxy core (radius $\lesssim 1$ $\mathrm{kpc}$ ). The kinematics of the old stellar population place 16 of 20 of our E+As on a trend parallel to the Faber-Jackson relation that is offset by $\sim 0.6$ mag in R. Eighteen of $20 \mathrm{E}+$ As have $\mathrm{v} / \sigma<1$. As the young stars in these systems evolve, the luminosity offset will disappear and the remnants will be pressuresupported systems that lie on the Faber-Jackson relation. Although Zabludoff et al. spectroscopically selected the most extreme E+A galaxies in the local volume, the sample is kinematically diverse: velocity dispersions range from $\lesssim 30$ $\mathrm{km} \mathrm{s}^{-1}$ to $\sim 200 \mathrm{~km} \mathrm{~s}^{-1}$ over a luminosity range of $\mathrm{M}_{\mathrm{R}}=-19$ to $-22+5 \log$ $h$. Combining these results with an estimate of the number of galaxies that experience an $\mathrm{E}+\mathrm{A}$ phase, we conclude that the $\mathrm{E}+\mathrm{A}$ phase of galaxy evolution is important in the development of a large fraction of spheroid-dominated galaxies over a wide range of luminosities and masses. Our kinematic observations, together with evidence that E+As have recently evolved from a vigorous star forming phase to a quiescent phase (e.g., Couch and Sharples, Caldwell et al.) and that many have tidal features consistent with disk-like progenitors (Zabludoff et al.), indicate that these galaxies are undergoing a transformation from gas-rich, star-forming, rotationally-supported, disk-dominated galaxies into gaspoor, quiescent, pressure-supported, spheroid-dominated galaxies.
\end{abstract}

\footnotetext{
${ }^{1}$ University of California Observatories/Lick Observatory, Board of Studies in Astronomy and Astrophysics, University of California, Santa Cruz, CA 95064; stuart@ucolick.org

${ }^{2}$ Astronomy Department, University of Texas at Austin, Mail Code C1400, Austin, TX 78712; gebhardt@astro.as.utexas.edu

${ }^{3}$ Steward Observatory, University of Arizona, 933 N. Cherry Street, Tucson, AZ 85721; azabludoff@as.arizona.edu,dzaritsky@as.arizona.edu
} 
Subject headings: galaxies: evolution — galaxies: interactions — galaxies: kinematics and dynamics — galaxies: stellar content

\section{Introduction}

One of the oldest questions in galaxy evolution is the evolutionary connection, if any, between spiral and elliptical galaxies. More specifically, is it possible to identify unambiguously a population of gas-poor, quiescent, pressure-supported, spheroid-dominated galaxies that were formerly gas-rich, star forming, rotation-supported, disky galaxies? The spectra of "E+A" galaxies" (Dressler \& Gunn 1983), which have a relatively young (A-type) stellar population but lack significant [OII] emission, indicate that these galaxies had high levels of star formation 1-2 Gyr ago (Couch \& Sharples 1987; Newberry, Boroson \& Kirshner 1990; Schweizer 1996) and that star formation has effectively stopped within that time. Thus, E+As may be a crucial link between gas-rich, star forming galaxies and quiescent galaxies. Some E+As have tidal features (Zabludoff et al. 1996; hereafter Z96) roughly consistent with simulations of the merger of two spiral galaxies (Toomre \& Toomre 1972), suggesting that E+As lie morphologically between the end of the Toomre merger sequence (Toomre 1977) and a relaxed spheroidal end-product.

While $\mathrm{E}+\mathrm{A}$ galaxies possess some spectroscopic and morphological signatures of an evolutionary transition, their internal kinematics are poorly understood - most known E+As lie at intermediate and high redshifts. As a result, it has been impossible to answer the most important questions concerning this seemingly major transition phase in galaxy evolution: Do the kinematics of E+As support the picture in which star formation is abruptly extinguished in otherwise normal disk galaxies or are they consistent with the galaxy merger models that form spheroids? If E+As are pressure-supported, then are their properties consistent with those of normal spheroidal galaxies? What is the luminosity range of galaxies that could have evolved through an E+A phase? With long-slit spectroscopy of a substantial sample of nearby E+As, we can address these issues for the first time.

The 21 E+As drawn from 11,113 galaxies in the Las Campanas Redshift Survey (LCRS; Shectman et al. 1992, 1996) by Z96 is the largest uniformly-selected sample of E+As within $z \sim 0.2$. These galaxies have strong Balmer absorption features (average equivalent width of

\footnotetext{
${ }^{4}$ The term "E+A" comes from their spectra, which contain absorption lines from an old stellar population similar to that of elliptical galaxies (E-type) in addition to a relatively young (A-type) stellar population. We use the $\mathrm{E}+\mathrm{A}$ designation for historical reasons only and note that the purely spectroscopic designation $\mathrm{K}+\mathrm{A}$ (e.g., Franx 1993) is more appropriate.
} 
$\mathrm{H} \beta, \mathrm{H} \gamma$, and $\mathrm{H} \delta>5.5 \AA$ ) and little if any [OII] emission (flux detections are at a level $\leq 2 \sigma$ ). The majority $(\sim 75 \%)$ of these E+As lie in the field, well outside rich cluster environments. In this paper, we determine how the fraction of A stars, the stellar velocity, and the stellar velocity dispersion vary along the major axis for these galaxies.

What triggers the starburst and subsequent cessation of star formation in $\mathrm{E}+\mathrm{A}$ galaxies are puzzles. Possible explanations include galaxy-galaxy mergers or interactions (Schweizer 1982; Lavery \& Henry 1988; Liu \& Kennicutt 1995; Schweizer 1996), interactions of galaxies with the intracluster medium (Dressler \& Gunn 1983; Bothun \& Dressler 1986), and galaxy harassment (Moore et al. 1998). Detections of tidal features (Schweizer 1996, Z96) and the discovery of E+As outside the cluster environment (Oegerle, Hill \& Hoessel (1991), Z96) suggest that mergers or interactions are indeed at least one way to produce E+As. To understand whether this mechanism is responsible for all E+A's, we must examine their stellar kinematics and their spatial distribution of young stars. If major mergers are the dominant mechanism responsible for $\mathrm{E}+\mathrm{A}$ formation, and merger remnants settle quickly to the virial plane (Mihos 1999), we should find that a large fraction of E+As are dynamically pressure-supported and lie within the region of parameter space - the Faber-Jackson relation (Faber \& Jackson 1976) — defined by normal spheroidal galaxies. In contrast, disk-like kinematics in E+As would support a model in which star formation is abruptly extinguished in otherwise normal disk galaxies. Franx (1993) finds strong rotation in one distant, cluster $\mathrm{E}+\mathrm{A}$, and Caldwell et al. (1996) report rotation in three E+As in the Coma cluster. Unfortunately, these studies were limited to cluster environments, the samples are small, and the criteria for selecting E+As differ among authors (cf. $\S 4$ ). We present kinematic results for a sample of uniformly and conservatively defined E+As that span a range of environments.

Complementing the kinematics, the spatial distributions of the young and old stellar populations further discriminate among formation models. For example, if E+As are otherwise normal late-type galaxies in which star formation is quickly extinguished, one expects little or no difference between the distributions of old and young stars. On the other hand, if mergers are occurring, simulations predict a high concentration of young stars toward the center because gas is driven inward after dissipating its orbital energy (Noguchi 1988; Barnes \& Hernquist 1991; Mihos, Richstone \& Bothun 1992; Mihos \& Hernquist 1996). Star formation in mergers and their remnants is not limited to the nucleus (Rieke et al. 1985; Wright et al. 1988; Schweizer 1996; Mihos \& Bothun 1988) as suggested by some numerical simulations, but it is centrally concentrated. Observations of several E+A galaxies in clusters suggest that young stars are present throughout the galaxy (Franx 1993; Caldwell et al. 1996). It is not known, however, if these trends are characteristic of all E+As, including those outside of rich clusters. With spatially-resolved spectroscopy spanning a wide range in wavelength, we can now separate the distributions and kinematics of the young and old 
stellar populations in the Las Campanas E+A sample.

If the $\mathrm{E}+\mathrm{A}$ phase does mark a transition from disk to spheroid, what range of spheroids can be produced? The velocity dispersions of normal ellipticals range from $\sim 50$ to $\sim 300 \mathrm{~km}$ $\mathrm{s}^{-1}$ (Davies et al. 1983; Bender \& Nieto 1990), but the distribution for E+As is unknown. In lower luminosity ellipticals rotational support is increasingly important (Bender \& Nieto 1990), but no test for this trend has been made with E+A galaxy samples. If E+As occupy only a limited part of the Faber-Jackson relation then they mark an interesting, but not necessarily significant, phase in the evolution of some galaxies. However, if we observe E+As over the range of luminosities and velocity dispersions occupied by normal ellipticals, then this brief phase may be part of the evolution of many galaxies. Examining the kinematic properties of this large, unbiased sample of E+As will help us determine not only whether the $\mathrm{E}+\mathrm{A}$ phase represents a transition between galaxy types, but also what fraction of galaxies are likely to have evolved through it.

A concern in the interpretation of $\mathrm{E}+\mathrm{A}$ spectra is the degree to which bursts of star formation may be cloaked by dust or missed by the often small physical radius probed by the spectroscopic fiber or slit. Smail et al. (1999) conclude, based upon observations at near infrared and radio wavelengths, that some distant "post-starburst" galaxies are actually dust-obscured starbursts. To determine whether significant star formation is enshrouded by dust in our E+A sample, two studies (Chang et al. 2001, Miller et al. in preparation) search for radio continuum emission, which can be interpreted as evidence for on-going star formation and is impervious to dust. Of the E+As in the Z96 sample, only two of the 17 observed to date are detected in radio continuum. Their detection limits of a few times $10^{21}$ $h^{-2} \mathrm{~W} \mathrm{~Hz}^{-1}$ correspond to upper limits of $0.5-1 h^{-2} \mathrm{M}_{\odot} \mathrm{yr}^{-1}$. Thus, we conclude that E+As are not disguised starbursts.

To better understand the properties and origins of E+As along the lines just described, we obtain and analyze long-slit spectra for the $21 \mathrm{E}+\mathrm{A}$ galaxies in the Z96 sample. In Section 2 we describe the data, our reduction procedure, and our methods for separating the kinematics and spatial distributions of young and old stellar populations. In Section 3 we present the results of our kinematic analysis. In Section 4, we discuss the implications of our results, and in Section 5 we present our conclusions. Throughout this analysis we assume a flat, $\Omega_{0}=1$ cosmology and $\mathrm{H}_{0}=100 \mathrm{~km} \mathrm{~s}^{-1} \mathrm{Mpc}^{-1}$, which results in an image scale of $\sim 1$ kpc per arcsec for the typical galaxy in our sample. 


\section{Data and Analysis}

\subsection{Observations and Reduction}

We obtained long-slit spectra of our sample of E+As using the du Pont 2.5-meter telescope at Las Campanas ${ }^{5}$ during November 1995, February 1996, and April 1996. We used the B\&C Spectrograph with the TEK-1 (1024 pixels $\times 1024$ pixels) CCD. Table 1 summarizes our observations: galaxy name and position (Columns 1-3), the total exposure time (Column 4), and the spectral range (Column 5). Galaxy names, EA 1 through EA 21, denote increasing strength of the $4000 \AA$ break spectral feature (cf. Z96). Galaxies with lowest numbers have the weakest break and thus have had the most recent and/or intense burst of star formation. For the remainder of this paper, we refer to each galaxy by its assigned number.

The data for this study consist of three to four coadded 1800 second exposures of each E+A galaxy using a 1.5-arcsecond slit. The pixel scale is 0.59 "/pixel in the spatial direction and $1.3 \AA /$ pixel in spectral direction. The spatial resolution, measured from stellar spectra, is $\sim 1.6^{\prime \prime}$, and typically varies by $\sim \pm 0.3^{\prime \prime}$ during each night. The spectral resolution, measured from the night sky lines, is 1.95 pixels full-width at half maximum (or $2.5 \AA$ ) and typically varies by $\sim 5 \%$ between observations. Because one of our principal goals is to examine both the older and younger stellar populations, the spectral range is selected to include both $\mathrm{Mg} I$ $5175 \AA$, which mostly originates from the older populations, and $\mathrm{H} \delta$, which mostly originates from the younger populations. The total spectral coverage is $\sim 1300 \AA$. The resulting spectra have a signal-to-noise $(\mathrm{S} / \mathrm{N})$ of $\sim 40$ per pixel for an aperture along the slit that includes the entire galaxy. In all cases, we aligned the slit with the major axis of the galaxy; for EA 20, we also obtained a minor-axis profile.

We apply standard $\mathrm{IRAF}^{6}$ routines and reduction techniques to the raw images. First, we subtract both an overscan region and an average bias frame from each image. Next, we divide the image by an image of a uniformly illuminated region of the dome interior. We obtain separate flat-field images for each grating angle that we use. We remove small-scale variations in sensitivity, and preserve the large-scale illumination function and slit function, by fitting and dividing the flats by a 2-dimensional function (25th order in the wavelength direction and 8th order in the spatial direction). We obtain spectra of twilight skies to

\footnotetext{
${ }^{5}$ Las Campanas observatory is operated by the Observatories of the Carnegie Institution of Washington.

${ }^{6}$ IRAF, the Image Reduction and Analysis Facility, is distributed by the National Optical Astronomy Observatories, which are operated by the Association of Universities for Research in Astronomy, Inc., under cooperative agreement with the National Science Foundation.
} 
Table 1. Observations

\begin{tabular}{|c|c|c|c|c|}
\hline Galaxy $^{\mathrm{a}}$ & RA & Dec & Total $\mathrm{t}_{\exp }(\mathrm{s})$ & Observed $\lambda$ range $(\AA)$ \\
\hline 1 & $10^{\mathrm{h}} 58^{\mathrm{m}} 48^{\mathrm{s}} .97$ & $-11^{\circ} 54^{\prime} 9^{\prime \prime} .80$ & 7200 & $4320-5630$ \\
\hline 2 & $2^{\mathrm{h}} 15^{\mathrm{m}} 43^{\mathrm{s}} .24$ & $-44^{\circ} 46^{\prime} 36^{\prime \prime} .70$ & 8100 & $4450-5760$ \\
\hline 3 & $12^{\mathrm{h}} 6^{\mathrm{m}} 31^{\mathrm{s}} \cdot 34$ & $-12^{\circ} 5^{\prime} 55^{\prime \prime} 40$ & 5400 & $4320-5630$ \\
\hline 4 & $3^{\mathrm{h}} 58^{\mathrm{m}} 23.42$ & $-44^{\circ} 43^{\prime} 40^{\prime \prime} \cdot 29$ & 4400 & $4490-5810$ \\
\hline 5 & $1^{\mathrm{h}} 56^{\mathrm{m}} 0.12$ & $-44^{\circ} 51^{\prime} 49^{\prime \prime} 0$ & 9000 & $4540-5850$ \\
\hline 6 & $11^{\mathrm{h}} 51^{\mathrm{m}} 21^{\mathrm{s}} .96$ & $-2^{\circ} 53^{\prime} 55^{\prime \prime} .9$ & 7200 & $4380-5680$ \\
\hline 7 & $22^{\mathrm{h}} 38^{\mathrm{m}} 17^{\mathrm{s}} .77$ & $-38^{\circ} 50^{\prime} 17^{\prime \prime} .0$ & 5400 & $4490-5810$ \\
\hline 8 & $14^{\mathrm{h}} 29^{\mathrm{m}} 20^{\mathrm{s}} .26$ & $-12^{\circ} 44^{\prime} 18^{\prime \prime} .20$ & 7200 & $4500-5810$ \\
\hline 9 & $1^{\mathrm{h}} 15^{\mathrm{m}} 24^{\mathrm{s}} .20$ & $-41^{\circ} 50^{\prime} 10^{\prime \prime} 69$ & 5400 & $4310-5620$ \\
\hline 10 & $2^{\mathrm{h}} 9^{\mathrm{m}} 44^{\mathrm{s}} .50$ & $-44^{\circ} 21^{\prime} 43^{\prime \prime} .20$ & 7200 & $4490-5810$ \\
\hline 11 & $1^{\mathrm{h}} 12^{\mathrm{m}} 34^{\mathrm{s}} .56$ & $-41^{\circ} 38^{\prime} 21^{\prime \prime} .79$ & 7200 & $4540-5850$ \\
\hline 12 & $12^{\mathrm{h}} 3^{\mathrm{m}} 25^{\mathrm{s}} .97$ & $-2^{\circ} 37^{\prime} 50^{\prime \prime} .59$ & 6000 & $4450-5760$ \\
\hline 13 & $11^{\mathrm{h}} 17^{\mathrm{m}} 21^{\mathrm{s}} .50$ & $-12^{\circ} 36^{\prime} 13^{\prime \prime} \cdot 30$ & 5400 & $4450-5760$ \\
\hline 14 & $13^{\mathrm{h}} 54^{\mathrm{m}} 20.95$ & $-12^{\circ} 12^{\prime} 10^{\prime \prime} 60$ & 3600 & $4380-5680$ \\
\hline 15 & $14^{\mathrm{h}} 38^{\mathrm{m}} 5^{\mathrm{s}} .59$ & $-6^{\circ} 27^{\prime} 4^{\prime \prime} .90$ & 7200 & $4540-5850$ \\
\hline 16 & $12^{\mathrm{h}} 17^{\mathrm{m}} 21^{\mathrm{s}} .44$ & $-5^{\circ} 57^{\prime} 22^{\prime \prime} .70$ & 5400 & $4380-5680$ \\
\hline 17 & $10^{\mathrm{h}} 11^{\mathrm{m}} 20.17$ & $-2^{\circ} 40^{\prime} 53^{\prime \prime} .0$ & 7200 & $4320-5630$ \\
\hline 18 & $0^{\mathrm{h}} 20^{\mathrm{m}} 18.81$ & $-41^{\circ} 50^{\prime} 15^{\prime \prime} 60$ & 5400 & $4310-5620$ \\
\hline 19 & $2^{\mathrm{h}} 5^{\mathrm{m}} 51^{\mathrm{s}} .66$ & $-45^{\circ} 35^{\prime} 2^{\prime \prime} .79$ & 5700 & $4320-5630$ \\
\hline 20 & $0^{\mathrm{h}} 36^{\mathrm{m}} 20.9$ & $-39^{\circ} 13^{\prime} 41^{\prime \prime} .50$ & 3600 & $4310-5620$ \\
\hline $20^{\mathrm{b}}$ & $0^{\mathrm{h}} 36^{\mathrm{m}} 20.9$ & $-39^{\circ} 13^{\prime} 41^{\prime \prime} .50$ & 3600 & $4310-5620$ \\
\hline 21 & $11^{\mathrm{h}} 12^{\mathrm{m}} 52^{\mathrm{s}} .65$ & $-6^{\circ} 28^{\prime} 51^{\prime \prime} .59$ & 7200 & $4450-5760$ \\
\hline
\end{tabular}

a Numbers identifying E+A galaxies are from Z96.

${ }^{\mathrm{b}}$ This spectrum was taken with the slit along the galaxy minor axis. 
correct for the slit illumination function in the object spectra. We derive the illumination pattern by fitting the sky spectra with a 7 th order function in the spatial direction at five different points along the wavelength axis. We do not correct the CCD response function in the wavelength direction. After removing the sensitivity and illumination variations, a number of pixels remain that are consistently discrepant from the surrounding pixels in all images. We identify these CCD defects interactively and interpolate over each group of bad pixels in the narrowest direction spanned by the pixels.

Defects are introduced into individual images by cosmic rays. We apply an algorithm similar to that described by Windhorst et al. (1994) to remove these. For each galaxy, the stack of 2D spectra (typically, three) are compared by examining the pixel values within a moving boxcar. We calculate the median in the boxcar and use the CCD noise characteristics to determine the expected standard deviation within the boxcar. We then compare the value of the pixel at the center of the boxcar among the images in the stack. All pixels greater than a threshold deviation (typically 12 to $15 \sigma$ ) from the boxcar median are replaced by the median. We visually examine the resulting images and tune the threshold deviation to ensure that no emission lines are removed. Finally, we average the image stack to produce the final image.

After removing cosmic rays, we complete the reduction process by establishing the wavelength scale, rectifying the images, and subtracting the sky flux. The reference spectra for wavelength calibration are helium-argon lamp images taken before and after each galaxy image. We use a two-dimensional fit (7th order spatially and spectrally) from the calibration spectra to wavelength calibrate and rectify each galaxy spectrum. Finally, we fit a line in the spatial direction to the sky on either side of the slit and subtract the sky spectrum. Due to the angular length of the long slit $(3.5 \mathrm{arcmin})$ and the small angular extent of the galaxies (several arcseconds), the edges of the frames provide uncontaminated sky spectra.

\subsection{Measuring Line-of-Sight Velocity Distributions and Stellar Populations}

The observed spectral range, which includes $\mathrm{H} \beta, \mathrm{H} \gamma, \mathrm{H} \delta, \mathrm{Mg} \mathrm{I}$, and the G-band, enables us to extract the velocity distributions of young and old stars. Although the different spectral features are primarily associated with either young or old populations, they do not arise exclusively in one or the other. It would be incorrect, for example, to measure the velocity distribution of the young stellar population using the Balmer lines, while ignoring the contribution of the older population to those lines. To disentangle the kinematics of the two stellar populations, we simultaneously fit the velocity profile and the relative contribution of different stellar components. Our two major concerns in the extraction of the 
line-of-sight velocity distribution (LOSVD) are including the appropriate stellar templates and optimizing the combined stellar templates in fitting the observed spectra. We discuss these in turn.

The library of template spectra must contain the range of stellar spectral types found in the galaxy and match the spectral resolution and wavelength range of the galaxy spectrum. Ideally, one would construct a large template library with the same instrumental configuration as used for the galaxy observation. However, because of the redshift difference between galaxies and template stars, we could not match the spectral coverage of local standard stars to that of the $\mathrm{E}+\mathrm{A}$ galaxies (which lie at $0.06 \lesssim z \lesssim 0.12$ ) without incurring problematic overhead. Instead, we use publicly available spectra of template stars from the Coudé Feed Spectral Library at KPNO (Leitherer et al. 1996) and convolve those spectra to match the characteristics of our observational configuration. The KPNO library contains 684 stars with a broad range of spectral types at $1.8 \AA$ FWHM resolution. We select a subsample of 22 stars representing $\mathrm{A}$ dwarfs and $\mathrm{G}$ and $\mathrm{K}$ dwarfs and giants. We experimented with a variety of different stars, and our final template subsample provides good overall type coverage without involving an exorbitant number of stars. Note that A giants are not included among the templates because their lifetimes $(<1 \mathrm{Myr})$ are extremely short relative to main-sequence A star lifetimes ( 1 Gyr; Bressan et al. (1993)).

In Figure 1, we plot representative template spectra. It is apparent from the figure that the most significant spectral differences are between the A-type stars and the other templates. We correspondingly separate the templates into two groups: a "young" population represented by the A dwarfs, and an "old" population represented by the G- and K-type templates. The presence of main sequence A stars, whose lifetimes are $\sim 1$ Gyr, and the lack of bluer stars in the $\mathrm{E}+\mathrm{A}$ spectra indicate that the most recent star formation event occurred $\sim 1$ Gyr ago. The main sequence stars comprising the older population have lifetimes from 4 to 10 Gyr. We refer to the two stellar populations as the young and old populations.

To prepare the template stars for their application, we transform each spectrum to the resolution and redshift of each galaxy spectrum. We convolve the spectra using a Gaussian (e.g., van der Marel \& Franx (1993)) with $\sigma^{2}=\sigma_{\mathrm{g}}^{2}-\sigma_{\mathrm{t}}^{2}$. The resolution of the galaxy spectrum is $\sigma_{\mathrm{g}}(1.1 \AA)$ and that of the templates is $\sigma_{\mathrm{t}}(0.9 \AA$ for the KPNO library).

We measure the LOSVD from a series of five apertures along the slit and from an aperture that contains all of the galaxy light. The five apertures include a central aperture that is 1 pixel wide, two apertures at intermediate radii that are adjacent to the central aperture and are 2 pixels wide, and two more distant apertures that are adjacent to the intermediate apertures and are 3 pixels wide (i.e., the most distant aperture on each side of the slit spans the region from 2.5 to 5.5 pixels [or $\sim 1.5$ to $3.3 \mathrm{kpc}$ ] from the center). Next, 


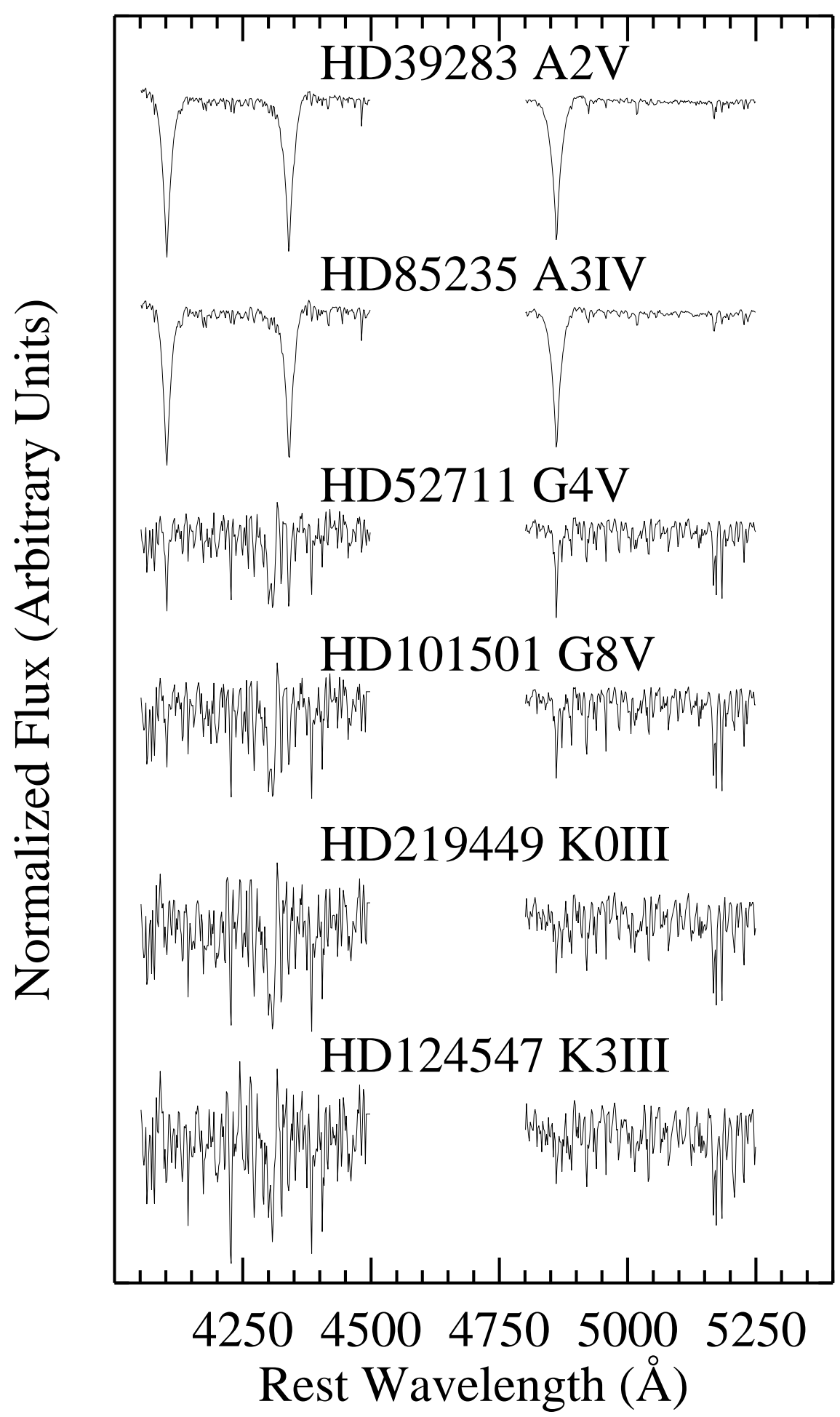

Fig. 1.- Representative template star spectra from Leitherer et al. (1996). Each spectrum is labeled with its spectral type. 
we fit and divide by the continuum in all template and galaxy spectra, alleviating the need for accurate flux calibration of our spectra.

We use a maximum likelihood procedure to estimate the best-fitting Gaussian LOSVD and template weights. We begin by choosing an initial velocity profile for each template type (young or old), which is characterized by the fractional contribution from each template star to the total light and by the Gaussian parameters (mean velocity and velocity dispersion) for that population. We convolve this line profile with a weighted-averaged template (the average of the template spectra where each star has its particular weight), subtract this spectrum from the galaxy spectrum, and calculate the residuals. We vary the template weights and velocity profile parameters (Gaussian coefficients) and identify the best match to the galaxy spectrum. Each template type is forced to have uniform kinematics, i.e., all of the young, A-type stars have the same LOSVD, but the contribution of each A star template to the total spectrum is allowed to vary. Rather than allowing the relative contributions of the populations to vary freely, we could have used evolutionary spectral synthesis codes (e.g., Bruzual \& Charlot (1993)) to model the contributions. However, because the integrated model spectra for populations of age $\lesssim 1 \mathrm{Gyr}$ are rather uncertain and because such a procedure introduces other unknowns such as the initial mass function, we prefer to fit the relative contributions of young and old stars to the spectrum.

We vary portions of this analysis to test our assumptions. For example, we add skew and kurtosis terms by replacing the Gaussian profile LOSVD profile with a Gauss-Hermite profile. We find no significant differences between the Gaussian and Gauss-Hermite fits, and thus we do not report the results for the Gauss-Hermite fits. We also compare the results from our standard method to those from a non-parametric fit (Gebhardt et al. 2001) and find that the two methods yield similar results. We conclude that deviations from a Gaussian are sufficiently insignificant that they do not adversely affect the results of the parametric fits.

We perform a Monte-Carlo analysis to measure the uncertainties in the recovered parameters. We generate 100 Monte Carlo realizations of the data in each aperture by adding random noise with the appropriate RMS to the best-fit spectrum (which is constructed from the best-fitting combination of templates and the LOSVD). Each realization is then analyzed as described above. From the distribution of 100 model parameters, the 16th and 84th percentile values determine the $68 \%$ confidence band. The average squared difference between the best-fitting model and the data provides the RMS. This approach does not account for systematic differences between the models and observations, such as template mismatches. For this same reason, we are unable to accurately determine uncertainties in the young star light fraction when the best-fitting value is zero, in which case we adopt a minimum error of 
0.1 .

\subsection{Spectral Fitting}

We present examples of the fitted spectra in Figures 2 through 4 . The observed spectrum of each galaxy, for the full $\left(\sim 6.6^{\prime \prime}\right)$ aperture, is plotted along with the best-fit model. The RMS difference between the fit and the data is labeled, along with the fraction of light from A-type (young) stars, above each spectrum.

An examination of the full set of spectra reveals a minor, but systematic trend: in the majority of cases, the best-fitting model has a deficit of $\mathrm{H} \delta$ absorption, the correct amount of $\mathrm{H} \gamma$, and an excess of $\mathrm{H} \beta$ absorption. The most likely cause of this discrepancy is the lack of an accurate flux calibration. Because we have divided the spectra of both galaxies and templates by their continua, and because the relative contributions of continuum and lines vary differentially for young and old templates across our wavelength range, we are diluting the absorption features differently in the templates than in the galaxies. For example, because the younger stars have more blue continuum than the older stars, we are artificially diluting the bluer Balmer lines and enhancing the redder lines when we divide the young star spectra by their continua. We could rectify this situation by having our algorithm fit the continuum as well as the line strengths (which would require flux calibration of both template and galaxy spectra), but given the small differences seen in the equivalent widths of the Balmer lines (cf. Figures 2-4) we conclude that the potential biases from the uncertainties

in the flux calibration are at least as large as those from the Balmer discrepancies. Other potential sources for the discrepancy include an incomplete template library and/or emissionline filling of the Balmer absorption lines. We have checked both of these possibilities and neither provides a satisfactory explanation. These small discrepancies between the fitted and observed spectra have an insignificant effect on the kinematics, which is the primary focus here.

As mentioned previously, to investigate the properties of each galaxy as a function of radius, we have also extracted spectra within several radial bins. In Figure 5, we show six examples of the fits in these radial bins, spanning a representative $\mathrm{S} / \mathrm{N}$ range, for the central aperture and the two radial apertures. The spectrum in the final panel, which comes from the outermost aperture in EA 19, is obviously too poor for a kinematic analysis. We make no attempt to derive kinematics for this spectrum or for any aperture with a lower $\mathrm{S} / \mathrm{N}$; this limit corresponds to $\mathrm{S} / \mathrm{N} \sim 10$ or RMS $\sim 0.1$ ( $10 \%$ of the continuum flux). However, our Monte-Carlo simulations demonstrate that the young star fraction is a significantly more robust measurement than the kinematics, and we retain these measurements for all five 


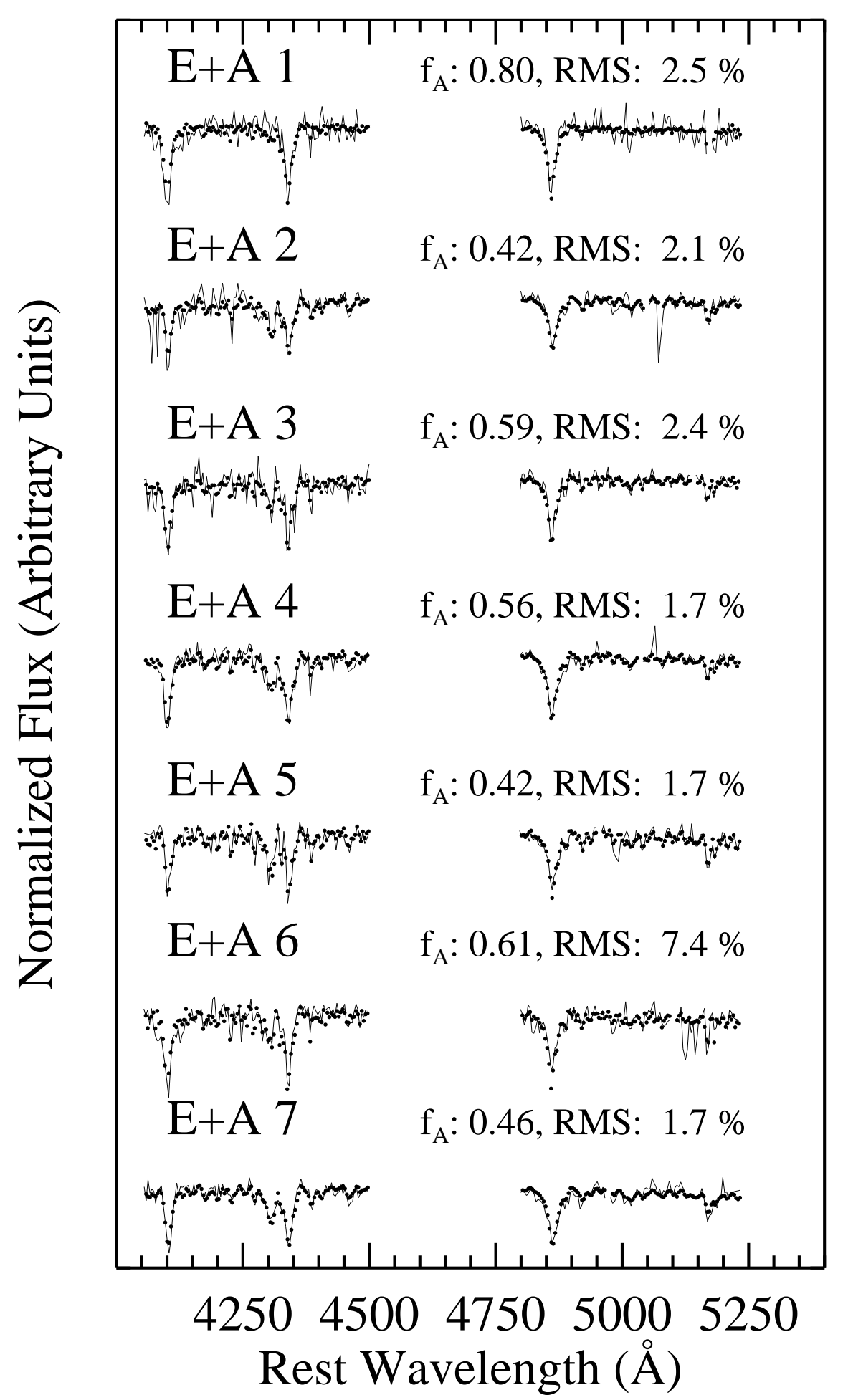

Fig. 2.- Observed spectra (solid lines) and our best fit (points). The RMS (per pixel) for each spectrum is calculated using the deviation between the continuum-normalized fit and data. 


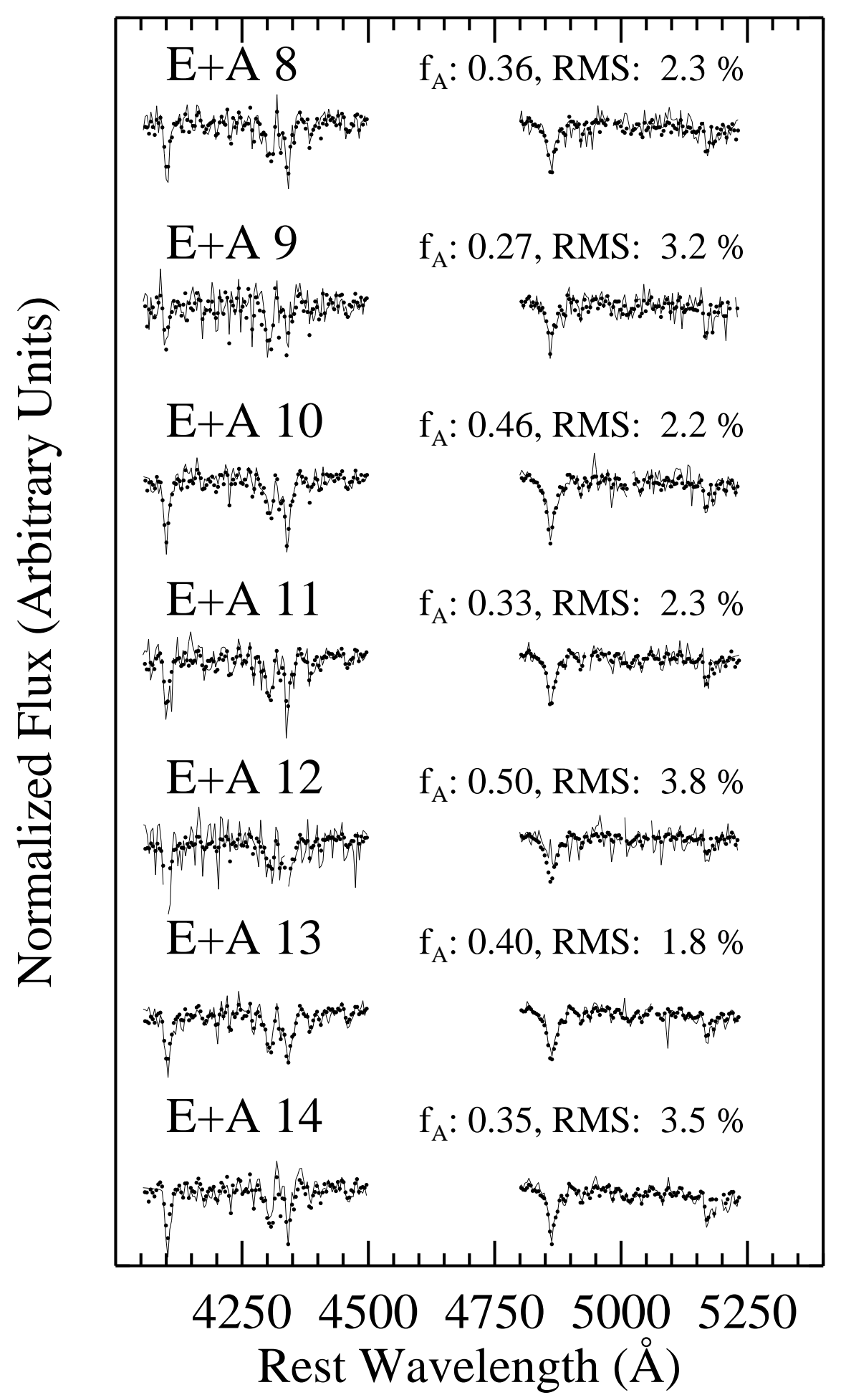

Fig. 3.- Observed spectra (solid lines) and our best fit (points). The RMS (per pixel) for each spectrum is calculated using the deviation between the continuum-normalized fit and data. 


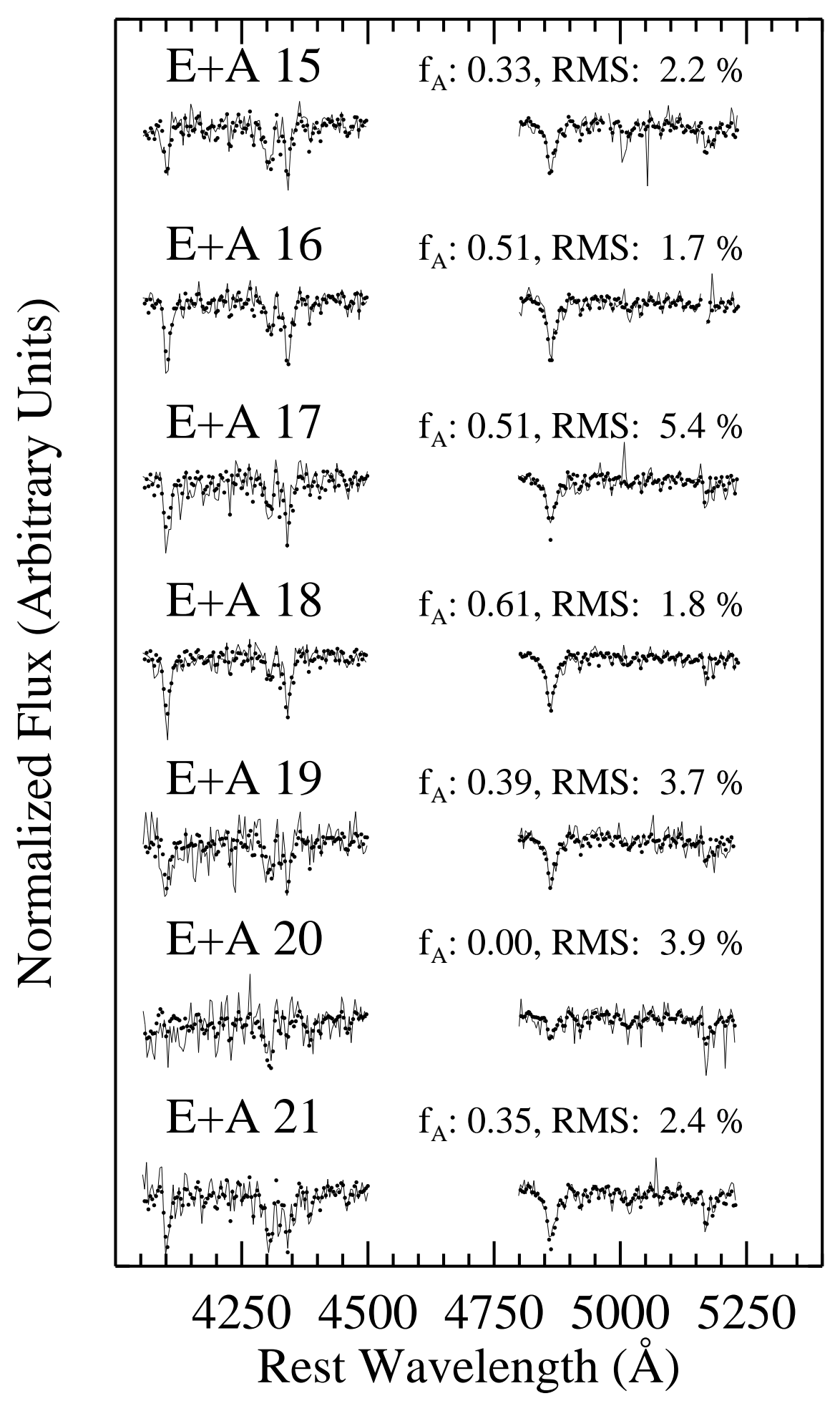

Fig. 4.- Observed spectra (solid lines) and our best fit (points). The RMS (per pixel) for each spectrum is calculated using the deviation between the continuum-normalized fit and data. 
apertures of each galaxy.

\section{Results}

For each aperture along the spectrum of each $\mathrm{E}+\mathrm{A}$, we measure the fraction of light contributed by the young, A-type stars (denoted by $\mathrm{f}_{A}$ ), the mean projected velocity relative to the galaxy center $\left(\left\langle\mathrm{v}_{\text {rot }}\right\rangle\right)$, and the velocity dispersion $(\sigma)$ (see Figures 6-11). Before examining the properties of the entire sample, we note three special cases. First, EA 20 shows no evidence for a young population in either a major or minor axis spectrum. We conclude that it was misclassified as an E+A due to noise in the optical LCRS fiber spectrum. We will exclude this galaxy from the rest of the discussion. Second, EA 12 has detectable emission in both $[\mathrm{OIII}]$ and Balmer lines. ${ }^{7}$ We exclude the central $\sim 9 \AA$ from each Balmer line in fitting the LOSVD for this galaxy. Despite the detected emission, the total [OIII] emission line equivalent width is $<1 \AA$ and the average absorption line equivalent width of $\mathrm{H} \beta, \gamma$, and $\delta$ is $\sim 5.5 \AA$. These parameters indicate that EA 12 does meet the selection criteria (cf. Z96), and we thus retain this galaxy in our analysis. Finally, HST imaging of EA 1 shows two distinct components that are separated spatially by $\sim 2.3 \mathrm{kpc}$ (Zabludoff et al. in preparation). We find that the differences between the kinematics of the two subcomponents are insignificant. In Figures 6-11 and in all future discussion we refer to the average of the two components as EA 1 . In Table 2 we list the value of $f_{A}$ for the aperture that includes the entire spectrum, the rotation velocity (see $§ 3.2 .1$ ), and velocity dispersion for each galaxy.

\subsection{Stellar population gradients}

The variation of $f_{A}$ across the slit measures the distribution of the recent star formation episode, which in turn constrains star formation and merger models (cf. Jog \& Solomon (1992); Mihos \& Hernquist (1996)). A trend is apparent in the behavior of $\mathrm{f}_{A}$ along the slit (Figures 6-11, top panels): the young star fraction typically peaks toward the center of the galaxy. Of the exceptions to this trend, EA 5, EA 7, EA 13, and EA 19 have a nearly constant $\mathrm{f}_{A}$, and EA 12 and EA 15 have a slight decline in $\mathrm{f}_{A}$ at the nucleus. For EA 12 at least, the nuclear minimum in $\mathrm{f}_{A}$ may be due to contamination by Balmer line emission, which is particularly prominent near the galaxy center. We have attempted to exclude this emission from our fits as described above, but some effects could remain.

\footnotetext{
${ }^{7}$ This galaxy is also one of two E+As in this sample detected in radio continuum by Miller et al. (2001, in prep.).
} 


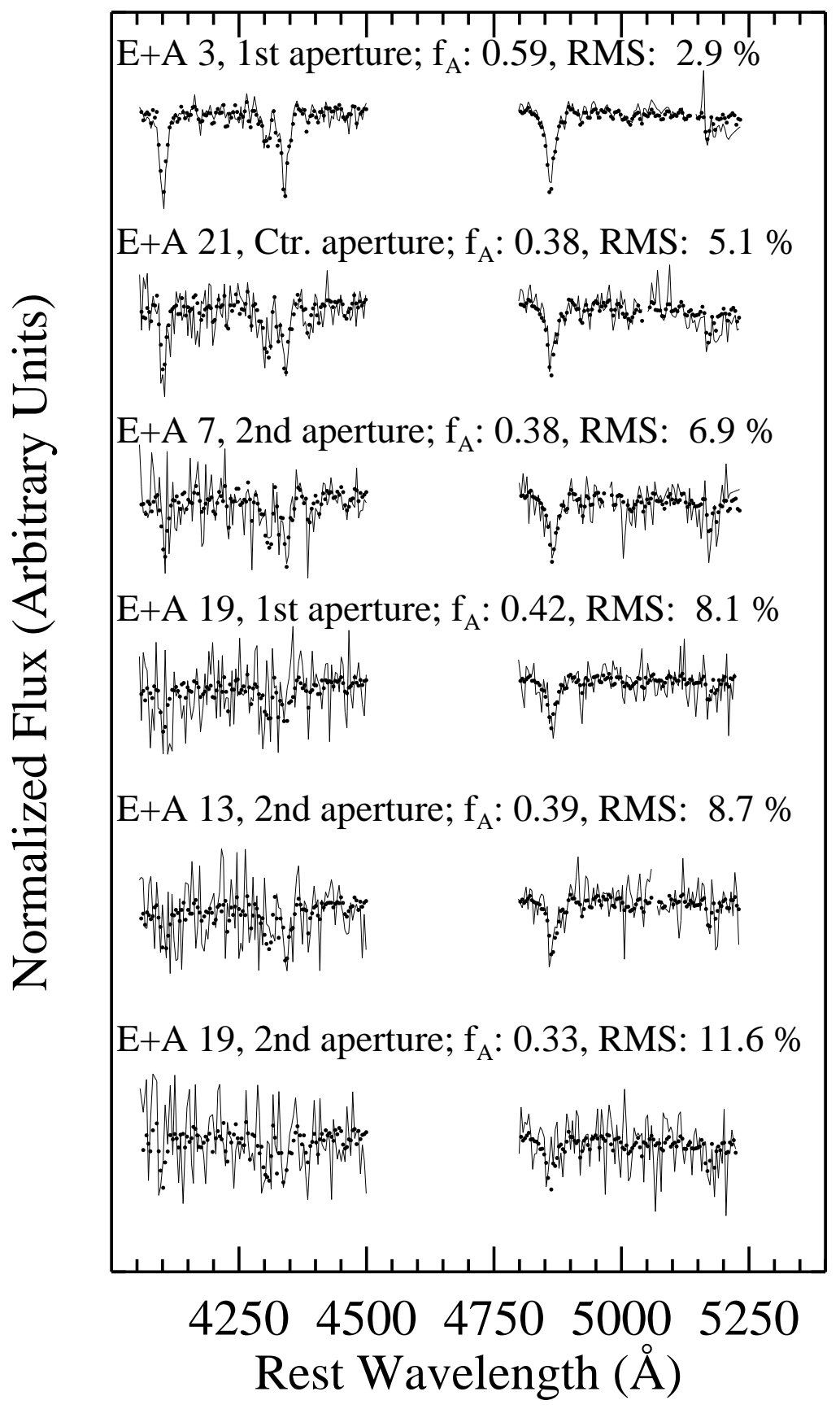

Fig. 5.- Representative spectral fits at various radii. From upper to lower panels these plots show: EA 3 at a radial distance of $0.9^{\prime \prime}(\sim 0.9 \mathrm{kpc})$, the central aperture for EA 21, EA 7 at a radial distance of $2.4^{\prime \prime}(3.4 \mathrm{kpc})$, EA 9 at $0.9^{\prime \prime}(0.8 \mathrm{kpc})$, EA 13 at $2.4^{\prime \prime}(2.9 \mathrm{kpc})$, and EA 19 at $2.4^{\prime \prime}(2.0 \mathrm{kpc})$. Note that the last spectrum is below our signal-to-noise cutoff (RMS $>10 \%)$ and is not used in the analysis. The RMS is calculated as in Figure 2. 


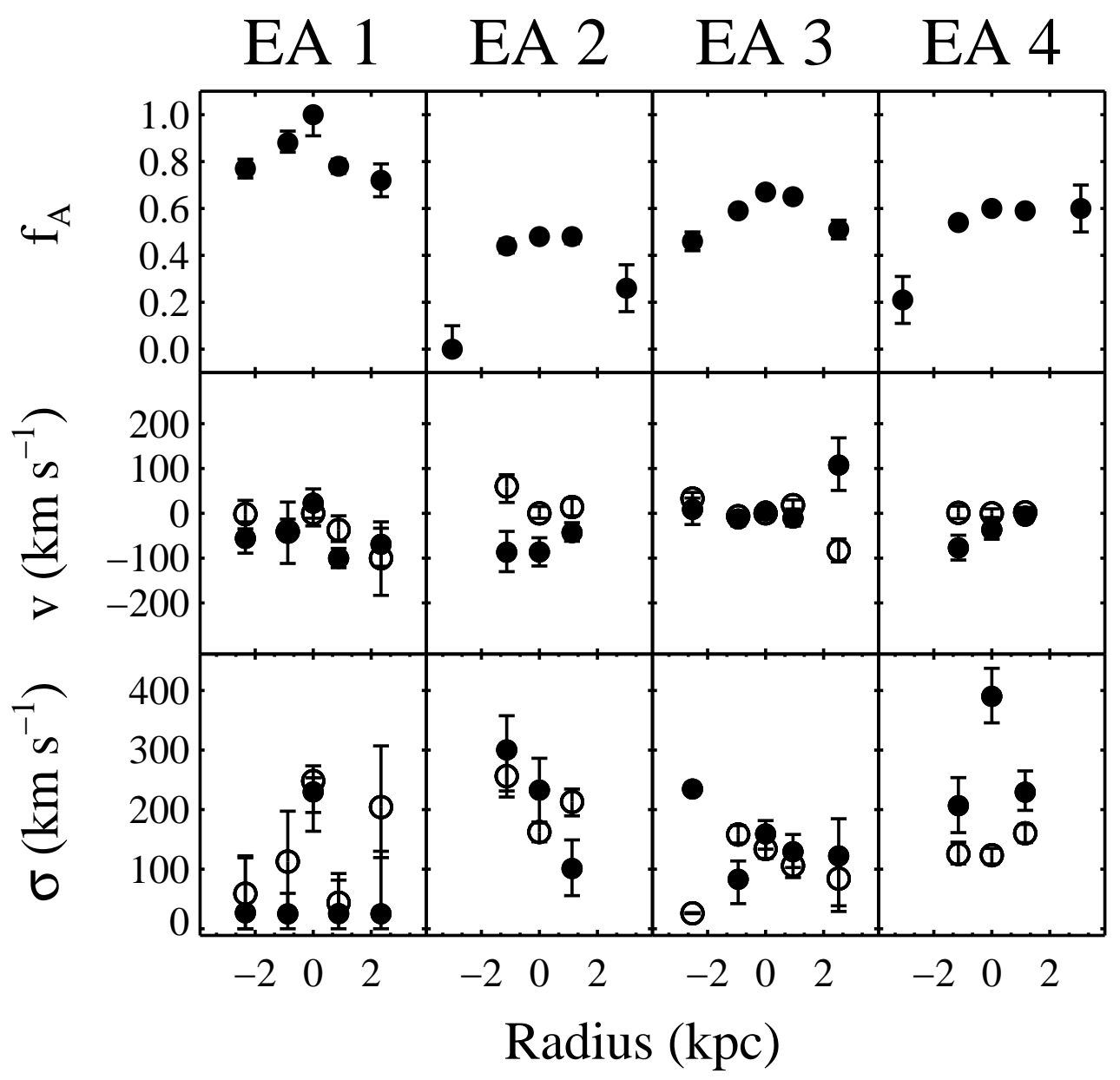

Fig. 6.- Fitted quantities for EA 1 to 4. Three measurements are plotted vs. radius. From upper to lower panels these quantities are (a) $f_{A}$, the A-type stellar fraction, (b) the mean velocity, and (c) the velocity dispersion. Open symbols represent the old stellar population, and filled symbols are the young population. The velocity and velocity dispersion are calculated only for the three central apertures unless the signal-to-noise in the outer apertures exceeds 10; however, we retain the measurements of A star fraction in the outer apertures (see text). 


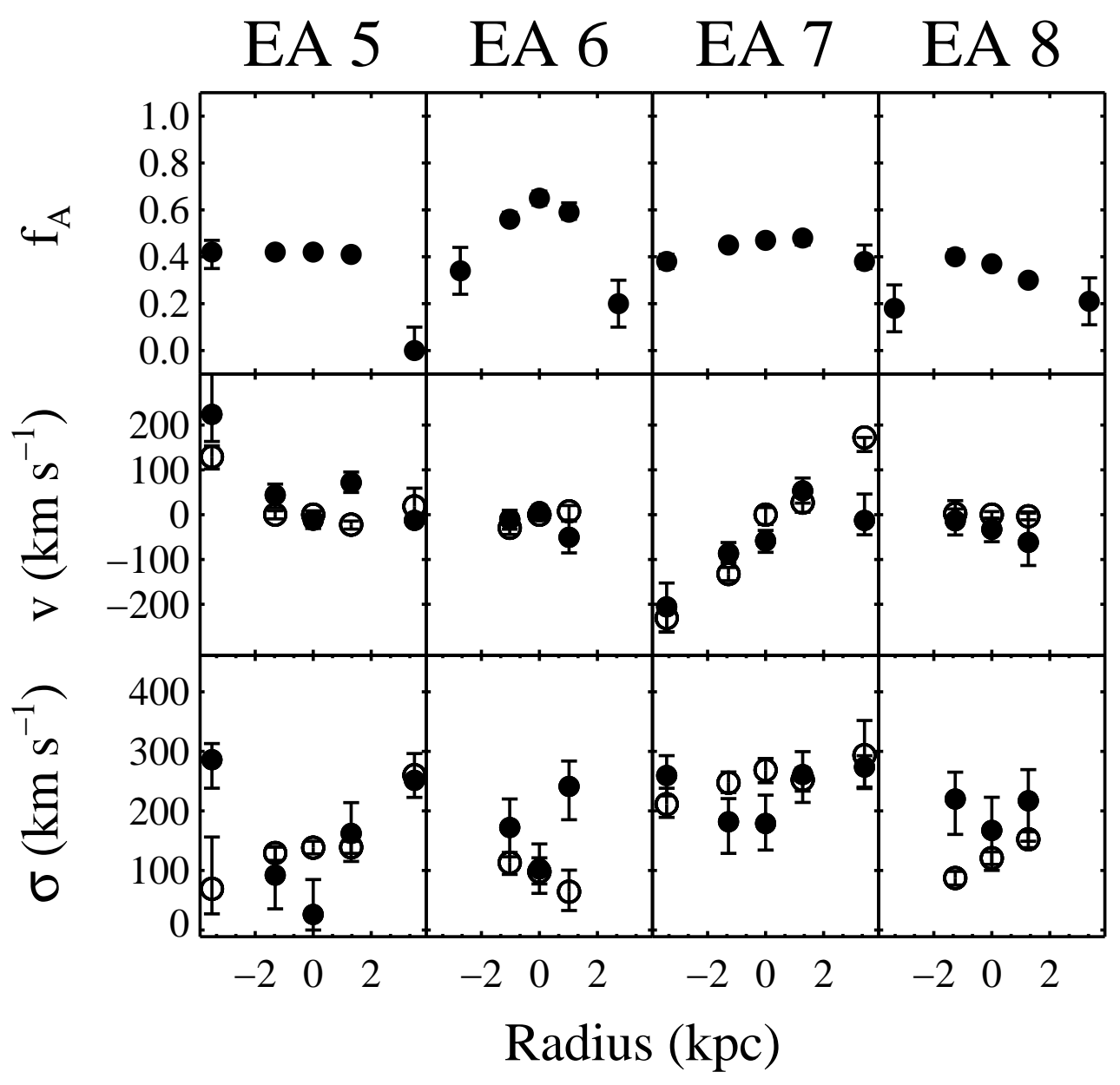

Fig. 7.- Same as Figure 6, but for EA 5 to 8. 


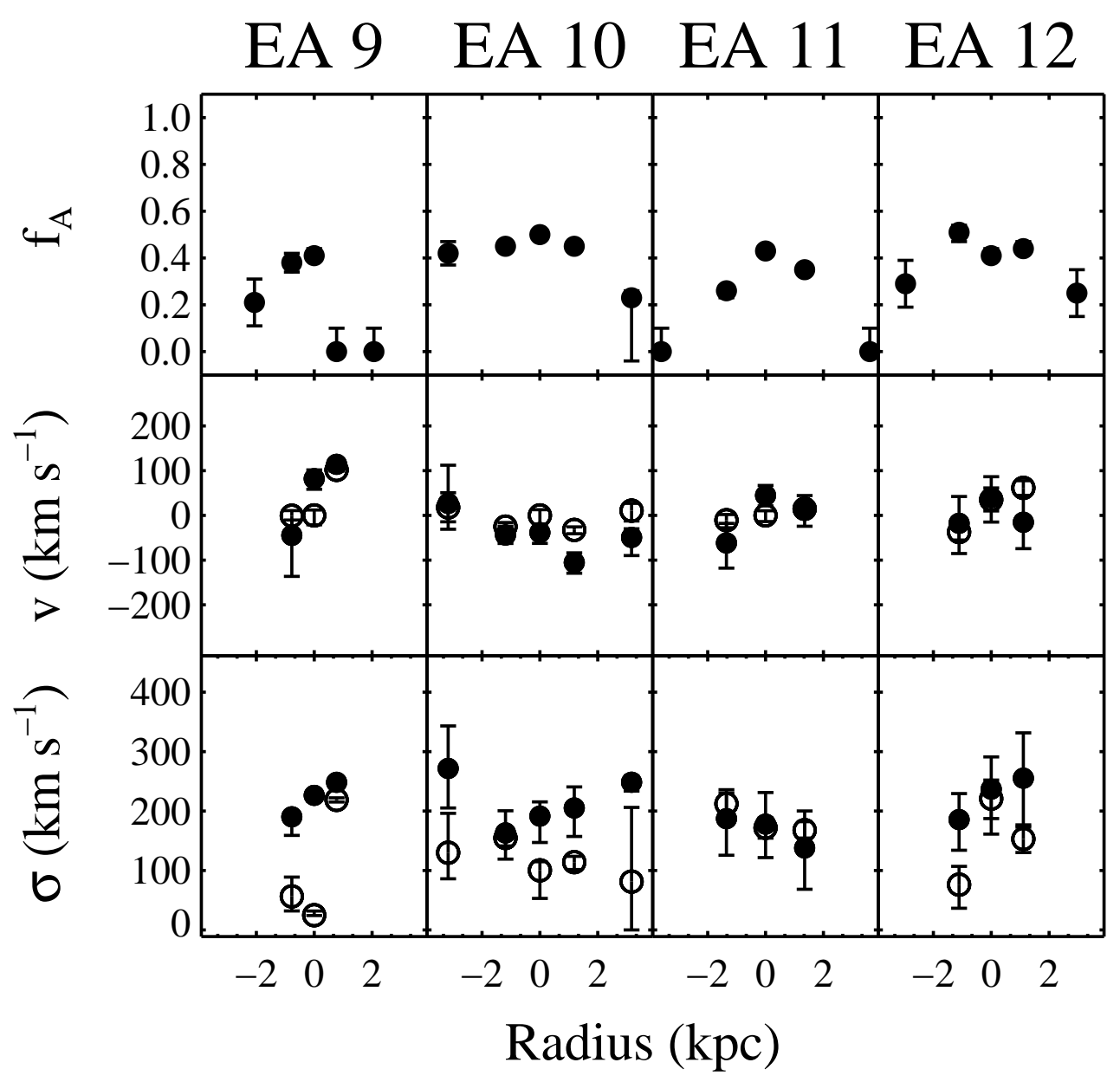

Fig. 8.- Same as Figure 6, but for EA 9 to 12 


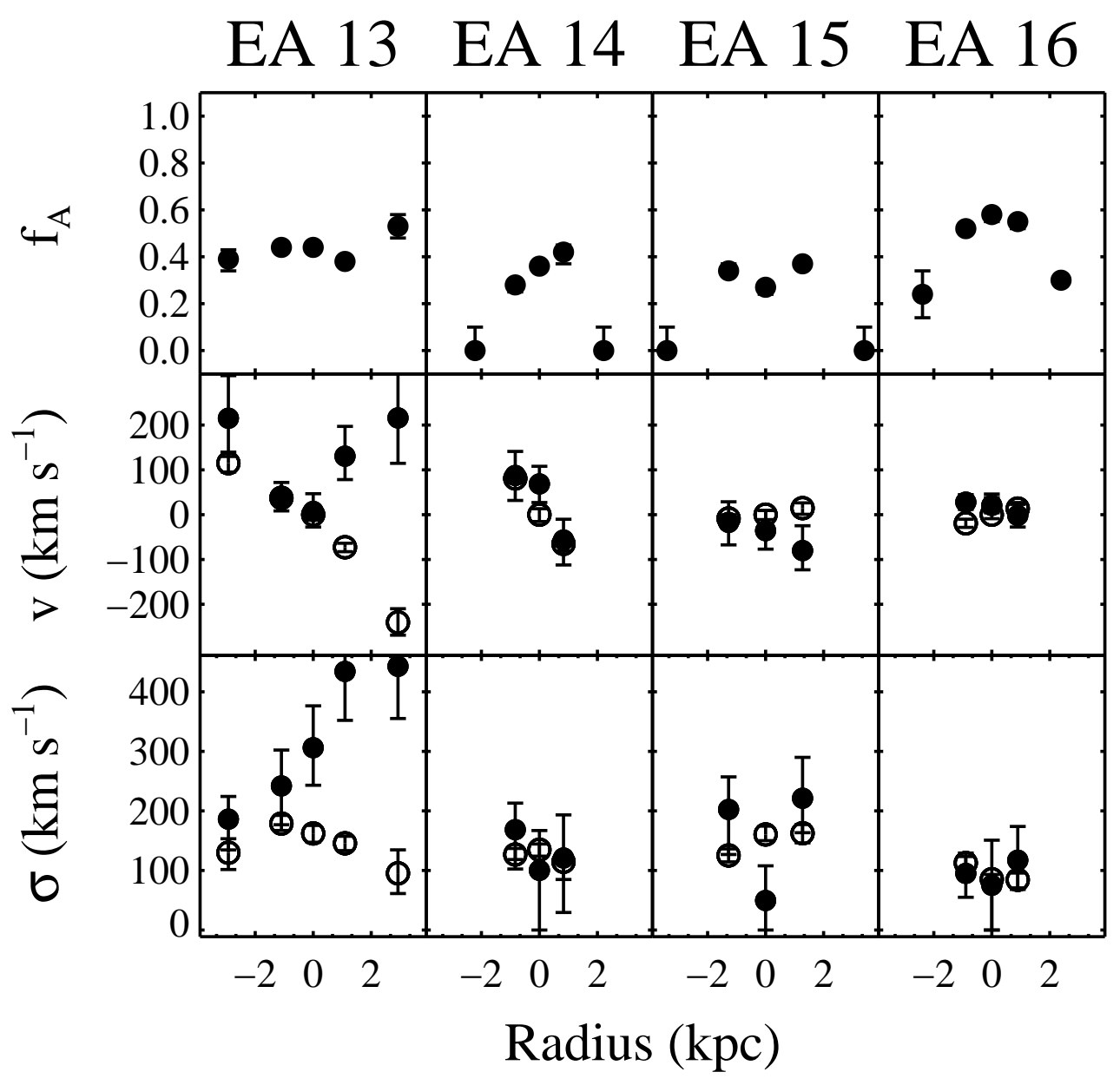

Fig. 9.- Same as Figure 6, but for EA 13 to 16. 


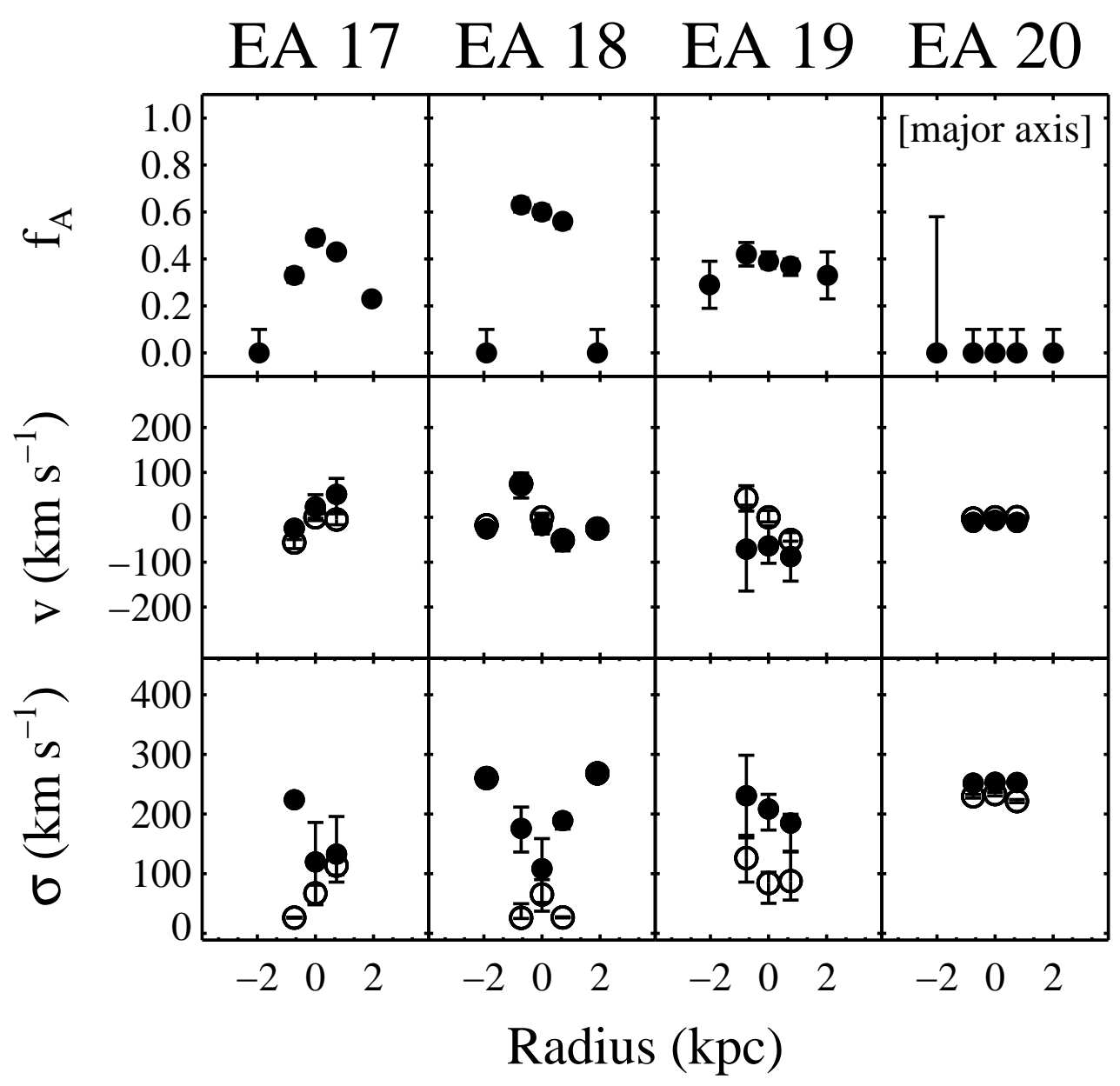

Fig. 10.- Same as Figure 6, but for EA 17 to 20. 


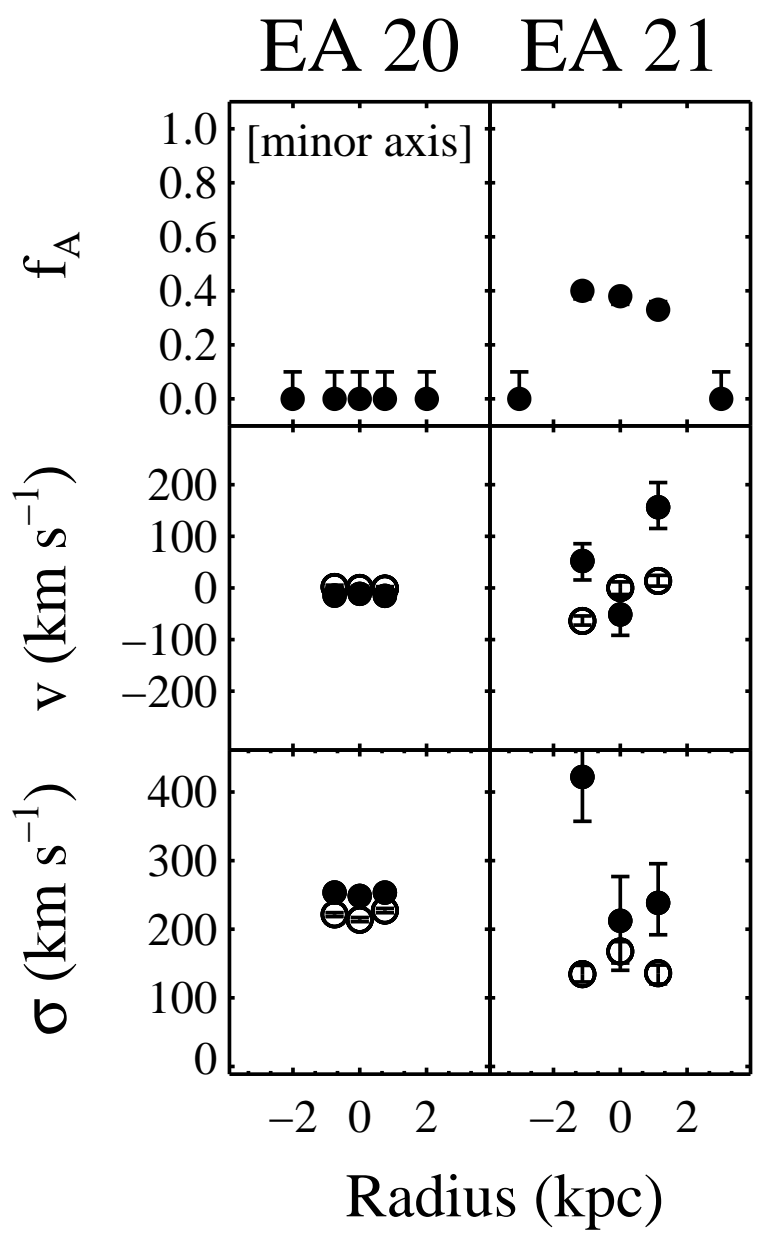

Fig. 11. - Same as Figure 6, but for the minor axis of EA 20 and for EA 21. 
Table 2. Galaxy Properties

\begin{tabular}{|c|c|c|c|c|c|c|c|}
\hline \multirow[t]{2}{*}{ Name } & \multirow[t]{2}{*}{$\mathrm{M}_{\mathrm{R}}^{\mathrm{a}}$} & \multirow[t]{2}{*}{$\mathrm{z}$} & \multirow[t]{2}{*}{$\mathrm{f}_{A}$} & \multicolumn{2}{|c|}{$\left\langle\mathrm{v}_{\text {rot }}\right\rangle\left(\mathrm{km} / \mathrm{s}^{-1}\right)$} & \multicolumn{2}{|c|}{$\sigma\left(\mathrm{km} \mathrm{s}^{-1}\right)^{\mathrm{b}}$} \\
\hline & & & & Old & Young & Old & Young \\
\hline EA 1 & -19.45 & 0.08 & $0.80_{-0.03}^{+0.03}$ & $24_{-54}^{+57}$ & $19_{-17}^{+17}$ & $35_{-35}^{+39}$ & $23_{-23}^{+53}$ \\
\hline EA 2 & -20.72 & 0.10 & $0.42_{-0.03}^{+0.02}$ & $23{ }_{-20}^{+16}$ & $22_{-25}^{+24}$ & $2022_{-16}^{+17}$ & $193_{-46}^{+32}$ \\
\hline EA 3 & -21.73 & 0.08 & $0.59_{-0.03}^{+0.02}$ & $23_{-23}^{+17}$ & $25_{-17}^{+18}$ & $120_{-20}^{+22}$ & $56_{-32}^{+35}$ \\
\hline EA 4 & -21.45 & 0.11 & $0.56_{-0.01}^{+0.01}$ & $1_{-9}^{+8}$ & $35_{-16}^{+15}$ & $131_{-9}^{+9}$ & $246_{-25}^{+24}$ \\
\hline EA 5 & -20.73 & 0.13 & $0.42_{-0.01}^{+0.02}$ & $33_{-25}^{+24}$ & $52_{-18}^{+34}$ & $120_{-8}^{+7}$ & $94_{-41}^{+34}$ \\
\hline EA 6 & -20.06 & 0.09 & $0.61_{-0.03}^{+0.02}$ & $18_{-13}^{+10}$ & $20_{-22}^{+20}$ & $23{ }_{-23}^{+1}$ & $158_{-18}^{+14}$ \\
\hline EA 7 & -22.40 & 0.12 & $0.46_{-0.01}^{+0.01}$ & $141_{-22}^{+20}$ & $83_{-18}^{+22}$ & $241_{-12}^{+11}$ & $208_{-31}^{+30}$ \\
\hline EA 8 & -20.45 & 0.12 & $0.36_{-0.01}^{+0.02}$ & $3_{-7}^{+7}$ & $24_{-36}^{+34}$ & $99_{-11}^{+8}$ & $141_{-55}^{+55}$ \\
\hline EA 9 & -18.74 & 0.07 & $0.27_{-0.26}^{+0.01}$ & $51_{-6}^{+19}$ & $79_{-17}^{+46}$ & $74_{-5}^{+134}$ & $172_{-60}^{+4}$ \\
\hline EA 10 & -20.53 & 0.11 & $0.46_{-0.02}^{+0.01}$ & $4_{-19}^{+21}$ & $34_{-17}^{+25}$ & $121_{-8}^{+7}$ & $183_{-41}^{+34}$ \\
\hline EA 11 & -20.64 & 0.13 & $0.33_{-0.03}^{+0.01}$ & $13_{-8}^{+10}$ & $38_{-29}^{+32}$ & $174_{-11}^{+12}$ & $95_{-46}^{+52}$ \\
\hline EA 12 & -20.30 & 0.10 & $0.50_{-0.03}^{+0.03}$ & $49_{-11}^{+12}$ & $1_{-42}^{+45}$ & $111_{-18}^{+17}$ & $470_{-78}^{+69}$ \\
\hline EA 13 & -21.20 & 0.10 & $0.40_{-0.01}^{+0.02}$ & $116_{-20}^{+20}$ & $22_{-38}^{+42}$ & $165_{-9}^{+10}$ & $392_{-59}^{+66}$ \\
\hline EA 14 & -20.40 & 0.07 & $0.35_{-0.02}^{+0.01}$ & $73_{-9}^{+9}$ & $72_{-36}^{+38}$ & $135_{-10}^{+10}$ & $73_{-73}^{+50}$ \\
\hline EA 15 & -20.30 & 0.12 & $0.33_{-0.02}^{+0.02}$ & $122_{-8}^{+8}$ & $32_{-37}^{+31}$ & $125_{-10}^{+9}$ & $187_{-57}^{+56}$ \\
\hline EA 16 & -19.87 & 0.08 & $0.51_{-0.01}^{+0.02}$ & $16_{-7}^{+8}$ & $15_{-15}^{+15}$ & $93_{-8}^{+7}$ & $95_{-29}^{+36}$ \\
\hline EA 17 & -19.05 & 0.06 & $0.51_{-0.04}^{+0.05}$ & $26_{-7}^{+9}$ & $38_{-21}^{+18}$ & $23{ }_{-23}^{+1}$ & $189_{-8}^{+9}$ \\
\hline EA 18 & -19.90 & 0.06 & $0.61_{-0.02}^{+0.02}$ & $33_{-9}^{+14}$ & $31_{-8}^{+8}$ & $23_{-23}^{+4}$ & $168_{-20}^{+16}$ \\
\hline EA 19 & -19.70 & 0.07 & $0.39_{-0.03}^{+0.02}$ & $46_{-16}^{+17}$ & $8_{-50}^{+56}$ & $84_{-34}^{+18}$ & $208_{-35}^{+25}$ \\
\hline EA 20 & -20.15 & 0.07 & $0.00{ }_{-0.00}^{+0.10}$ & $2_{-2}^{+2}$ & $\ldots$ & $204_{-2}^{+2}$ & $\ldots$ \\
\hline EA $20^{c}$ & -20.15 & 0.07 & $0.00_{-0.00}^{+0.10}$ & $2_{-2}^{+2}$ & & $200_{-2}^{+2}$ & \\
\hline EA 21 & -20.22 & 0.10 & $0.35_{-0.02}^{+0.02}$ & $39_{-7}^{+7}$ & $52_{-27}^{+30}$ & $134_{-9}^{+7}$ & $237_{-45}^{+48}$ \\
\hline
\end{tabular}

${ }^{a}$ Magnitudes are from the LCRS survey (Lin et al. 1996); errors are $\sim 0.1$ magnitude.

${ }^{\mathrm{b}} \sigma=23 \mathrm{~km} \mathrm{~s}^{-1}$ is the limit of our resolution; all entries with $\sigma=23 \mathrm{~km}$ $\mathrm{s}^{-1}$ are upper limits.

${ }^{\mathrm{c}}$ Slit aligned with minor axis. 
The second moment of the luminosity distributions measures the relative extent of the populations. Because our observations span angular distances of only $\sim 2^{\prime \prime}$ to $7^{\prime \prime}$ (or $\sim 2$ to 7 $\mathrm{kpc}$ ) across the galaxy, a range which is roughly comparable to the seeing (typically $1.5^{\prime \prime}$ ), a serious concern is that the light of the young stars that we observe outside the nuclear region is simply scattered nuclear light. To address this concern, we compare the distribution of light from the stellar populations of each galaxy with the seeing profile in Figure 12. The seeing profile is obtained from stellar spectra that were taken within a couple of hours before and after each galaxy spectrum. We overplot a Gaussian profile of the corresponding width (which ranges from 1.2" to 1.7" FWHM) for each galaxy in Figure 12.

We find that 6 of the $20 \mathrm{E}+\mathrm{A}$ galaxies have flux from young stars that is more than $2 \sigma$ above the Gaussian profile at both of the outermost measured points (EA 1, 3, 7, 12, 13, and 19). For comparison, the identical analysis for the old stellar population flux shows that 15 of the galaxies display a $2 \sigma$ flux excess relative to a nuclear source at the outermost radii. The remaining galaxies, EA 5, 6, 10, 15, and 21, also show old stellar populations that are more extended than the Gaussian, but not at the $2 \sigma$ level.

To quantify these results further, in Figure 13 we plot the second moment, $\zeta$, of each galaxy's luminosity distribution. For a normal distribution, $\zeta$ is the FWHM of the luminosity distribution divided by 2.35. Two vertical lines mark the range of $\zeta$ determined from the seeing profiles over the course of our observations. About one half of the young stellar populations are marginally resolved while the old populations are well resolved. Seven E+As (EA 1, 3, 7, 8, 12, 13, and 19) have resolved, extended young populations, with $\zeta$ exceeding the seeing by more than $40 \%$. Only one of the $20 \mathrm{E}+\mathrm{A}$ galaxies, EA 12, has a detectable young stellar population that is (marginally) more extended then its older population, but its central emission may have some effect on this result.

From our study of the stellar population distributions, we conclude that (1) the older stellar populations in these galaxies are more radially extended than the young stellar populations and (2) the recent star formation in E+A galaxies, though it is relatively centrally concentrated, is not always confined to the galaxy's central kiloparsec.

\subsection{Velocity Distributions}

Stellar velocity distributions are one of the fundamental differences between different galaxy types. In this Section we present and discuss our measurements of the first and second moments of the velocity distributions of $\mathrm{E}+\mathrm{A}$ galaxies. 


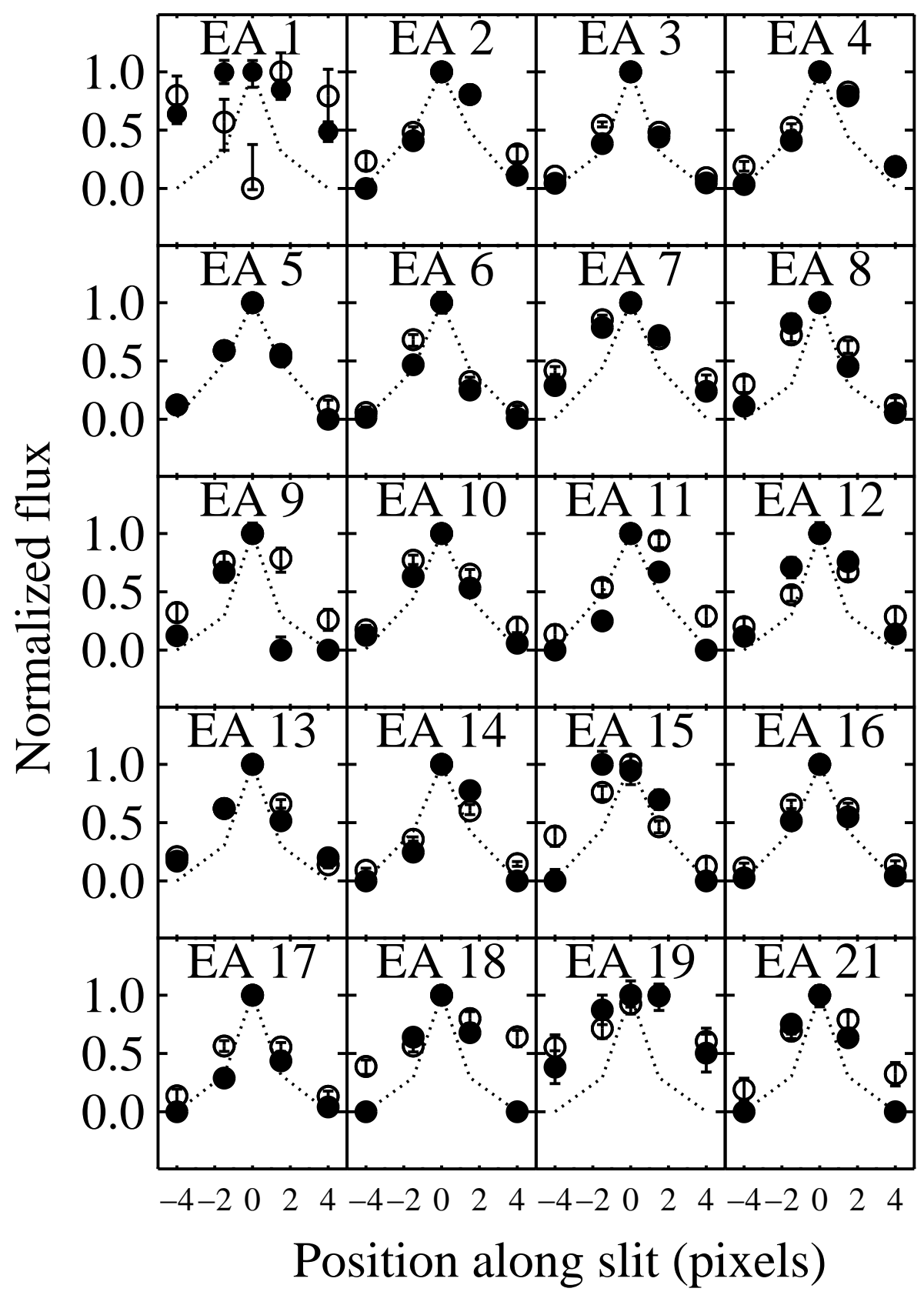

Fig. 12.- Flux contributed by each population as a function of radius, normalized at the central pixel. The filled circles represent the young stellar population, and the open circles are the older population. The dotted line represents a Gaussian seeing profile measured from a corresponding stellar image (see text). 


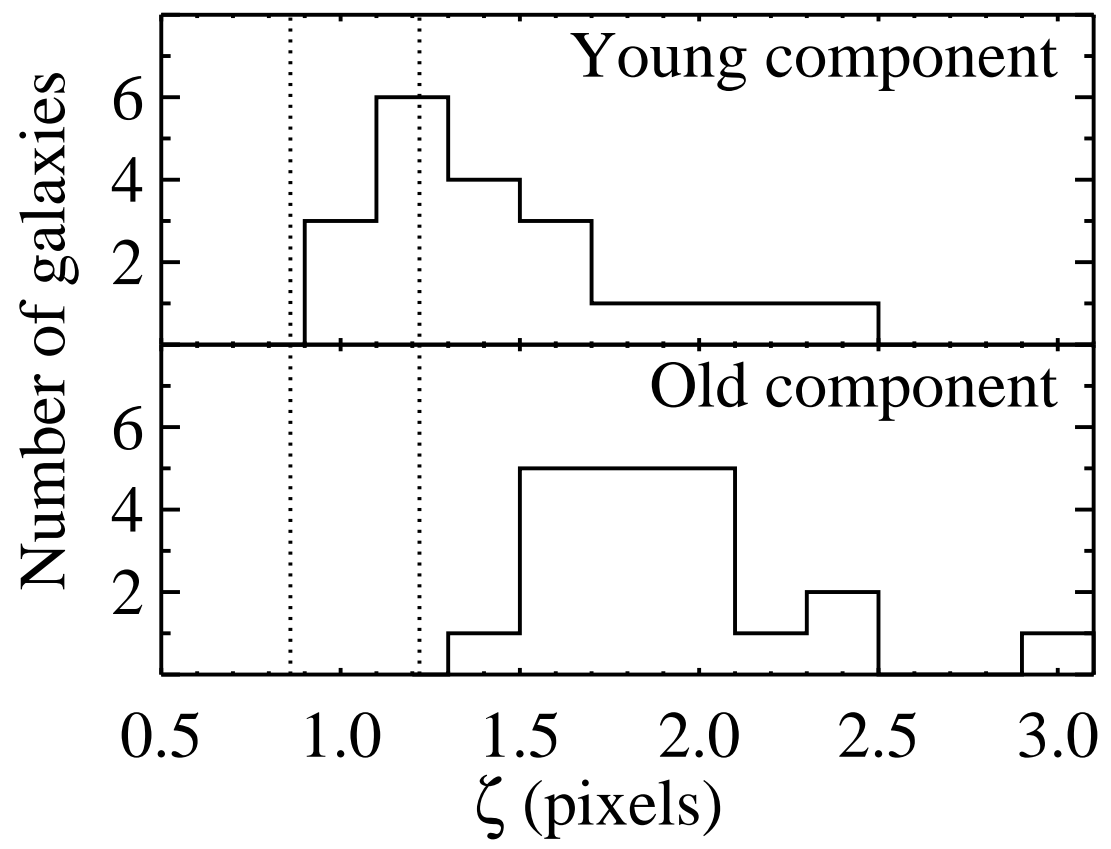

Fig. 13.- Histogram of the second moment of the luminosity distribution $(\zeta)$ for the young and old stellar populations. The dotted lines mark $\zeta$ for a Gaussian point spread function with the maximum and minimum seeing widths observed during the run. 


\subsubsection{Mean Velocities}

We begin by examining the first velocity moment (mean velocity) as a function of position along the slit. Monotonic variations along the slit would indicate rotation. It must be noted that these data are only able to probe rotation at distances of $\sim 1-3 \mathrm{kpc}$, near the galaxy centers.

From Figures $6-11$ it is evident that there is generally no strong rotation, in either the old or young populations of these galaxies. The most notable exception is EA 7, in which both stellar populations rotate at $\sim 200 \mathrm{~km} \mathrm{~s}^{-1}$ at a radius of $\sim 4 \mathrm{kpc}$. There is a suggestion of rotation in a few others, such as the old stellar populations of EA 13 and 14 and the young populations of EA 9 and 14. However, aside from these few exceptions, we find little evidence for significant rotation in the E+As.

We define the characteristic rotation velocity $\left\langle v_{\text {rot }}\right\rangle$ of a galaxy to be equal to one half the velocity difference across the slit. We average the velocities in the two radial bins on each side of the slit for higher signal-to-noise. The resulting rotation velocity is not directly comparable to the full rotation at the rotation curve turnover radius. For a solidbody rotation curve over the radial range probed, $\left\langle\mathrm{v}_{\text {rot }}\right\rangle$ would be the velocity at the average distance of our intermediate radial bins, $\sim 1.9 \mathrm{kpc}$. For reference, typical spiral galaxies have $\left\langle\mathrm{v}_{\text {rot }}\right\rangle \sim\left(70\right.$ to $\left.140 \mathrm{~km} \mathrm{~s}^{-1}\right) / \sin i$ (when measured at the same radius; see e.g., Héraudeau et al. (1999)), where $i$ is the unknown inclination angle.

Only six of the twenty $\mathrm{E}+\mathrm{As}$ in our sample have $\left\langle\mathrm{v}_{\text {rot }}\right\rangle>50 \mathrm{~km} \mathrm{~s}^{-1}$ for either the old or the young stellar populations (Table 2). Using a Kolmogorov-Smirnov (K-S) test, we find that the distributions of $\left\langle\mathrm{v}_{\text {rot }}\right\rangle$ of the young and old stellar populations are not significantly different. Not only are the distributions similar, but, for most of the E+As, rotation is observed either in both populations or not at all. For example, only four E+As have old stellar populations with $\left\langle\mathrm{v}_{\text {rot }}\right\rangle>50 \mathrm{~km} \mathrm{~s}^{-1}$ : EA 7, EA 9, EA 13, and EA 14. Of these four galaxies, three (EA 7, 9, and 14) also show evidence for rotation in the young stellar component. Only two galaxies (EA 7 and 13) have differences larger than $50 \mathrm{~km} \mathrm{~s}^{-1}$ in $\left\langle\mathrm{v}_{\text {rot }}\right\rangle$ between the young and old populations. However, interpreting the results from such comparisons, whether they are similarities or differences, is complicated by the different spatial distribution of the two populations.

An alternative explanation for the monotonic change in velocity across the slit is bulk relative motion of two merging galaxies. However, none of the rotating E+As show separated optical components on the Digitized Sky Survey images (see Z96), and only one of the five E+As for which we have HST imaging (EA 1; Zabludoff et al. in preparation) has two components (and EA 1 shows no apparent rotation). 
The majority of our sample does not appear to be rapidly rotating. Two factors may lead us to underestimate the degree of rotation in our sample. First, our rotation velocities must be corrected by $\sin i$ to account for inclination effects. However, it is unlikely that many of the galaxies would be fortuitously aligned close to face-on. Assuming a random distribution of inclinations, such that the probability of observing a galaxy with inclination $i<\theta$ is $1-\cos (\theta)$, a random distribution of galaxy velocities between 40 and $100 \mathrm{~km} \mathrm{~s}^{-1}$ a very conservative estimate - would result in a $\left\langle\mathrm{v}_{\text {rot }}\right\rangle$ distribution that has $\sim 25 \%$ of the measurements below $40 \mathrm{~km} \mathrm{~s}^{-1}$. In our sample, $75 \%$ of the galaxies have velocities below this threshold, suggesting that the rotation velocities of the E+As are smaller than the 40 to $100 \mathrm{~km} \mathrm{~s}^{-1}$ range. Second, poor spatial resolution may be a concern. If $\mathrm{E}+\mathrm{A}$ galaxies were rotationally supported only outside the central $\sim 2 \mathrm{kpc}$, we would not be able to observe that here. It may be significant that we do detect rotation in some of the galaxies for which our radial coverage is greatest (EA 3, EA 7). Although we cannot rule out rotation at larger radii than we are currently able to probe, $\left\langle\mathrm{v}_{\text {rot }}\right\rangle \sin i$, even at the small radii we observe, is 70 to $140 \mathrm{~km} \mathrm{~s}^{-1}$ for a typical disk galaxy (Héraudeau et al. 1999) - much larger than what we observe in the $\mathrm{E}+\mathrm{A}$ sample.

From the rotation analysis, we conclude that (1) both rapidly rotating systems (cf. Franx (1993) and discussion above) and slowly or non-rotating systems can be found in the E + A phase, (2) most E+A galaxies in our sample (14/20) show no evidence for rotation and that for two other galaxies the evidence for rotation is marginal, and (3) the rotation (or lack thereof) is generally present in both the old and young stellar populations of an E+A galaxy.

\subsubsection{Velocity Dispersions}

The lack of rotation in most of the E+As in our sample indirectly suggests that most are pressure-supported. To assess this possibility, we measure the velocity dispersion, $\sigma$, and compare the dispersions of the young and old stellar populations. Differences between the two populations may arise if they have different spatial distributions, if one or both stellar populations are not yet in equilibrium after the hypothesized merger event, or if they have reached different dynamical equilibria.

In Figure 14, we present a histogram of $\sigma$ calculated from the "total galaxy" apertures of $\sim 7 \mathrm{kpc}$ in full width. Because this measure includes line-broadening due to rotation, the use of a "total" aperture is justified only for the low rotational velocities measured in the majority of these systems ( $\sigma$ will be somewhat inflated in systems with significant rotation, $\left.\left\langle\mathrm{v}_{\text {rot }}\right\rangle\right\rangle$ $\left.50 \mathrm{~km} \mathrm{~s}^{-1}\right)$. A K-S test indicates that the $\sigma^{\prime}$ s for the young and old populations are unlikely 
$(<1 \%$ chance) to come from the same parent distribution. To conservatively quantify the difference between the two populations, we first exclude the four lowest-luminosity E+As, for which the old populations' velocity dispersions are extremely low. We then calculate that the mean values of $\sigma$, and the errors in the means, are $190 \pm 12 \mathrm{~km} \mathrm{~s}^{-1}$ for the young population, and $130 \pm 5 \mathrm{~km} \mathrm{~s}^{-1}$ for the old population. Although the difference between $\sigma$ in the two populations is significant and argues that the younger component is more pressuresupported, it may be due to the aforementioned spatial differences between the populations. Because the young stars are more centrally concentrated, and $\sigma$ for a typical elliptical is larger toward the center (e.g., Davies (1981)), the larger velocity dispersions for the young stars may be a result of their position within the galaxies. In normal ellipticals, varying the aperture from the central kiloparsec outward can decrease the observed velocity dispersion up to $25 \%$, although the typical decline is 5 to $10 \%$ (Davies 1981; Gebhardt et al. 2000). From these data, we are unable to determine whether the observed velocity dispersion differences are caused by spatial or dynamical differences.

To compare the properties of the E+As to those of "normal" ellipticals, we draw a comparison sample from the studies of Faber et al. (1989) and Jørgensen \& Franx (1994). The Faber et al. data are published in the B-band, which we transform to R using a typical B-R color for this sample of 1.25 (determined from a comparison of galaxies that are in both the Faber et al. and the R-band Jørgensen \& Franx data). In Figure 15, we plot the FaberJackson (FJ) relation (Faber \& Jackson 1976) for these normal ellipticals and compare it to the FJ relations produced using either the kinematics of the young or old stellar populations of $\mathrm{E}+$ As. The $\mathrm{R}$ magnitudes are for the entire $\mathrm{E}+\mathrm{A}$ galaxy and are on a Gunn/Kron-Cousins hybrid system used by the LCRS group (Lin et al. 1996), who report that the differences between the standard and hybrid Kron-Cousins systems are small ( $\lesssim 0.1$ magnitude). For comparison with the literature data, we have adjusted the isophotal magnitudes of the $\mathrm{E}+\mathrm{A}$ galaxies to approximate total magnitudes by using the systematic trends between the LCRS isophotal magnitudes and the Sloan Digital Sky Survey Petrosian magnitudes, the latter of which are approximately total magnitudes (Blanton, M. R. et al. 2001).

There is no significant correlation between galaxy luminosity and the velocity dispersion of the younger populations in E+As. Nevertheless, with the exception of a few outliers (EA 12 and 13, which have extremely high velocity dispersions, and EA 1 and EA 3, with anomalously low velocity dispersions), the distribution of points is consistent with the FaberJackson relationship. All other galaxies are within the envelope formed by the "normal" ellipticals, although the scatter is visibly much larger than that for the "normal" ellipticals.

On the other hand, the old stellar populations have a significant correlation between galaxy luminosity and velocity dispersion (Spearman rank-order correlation coefficient of 


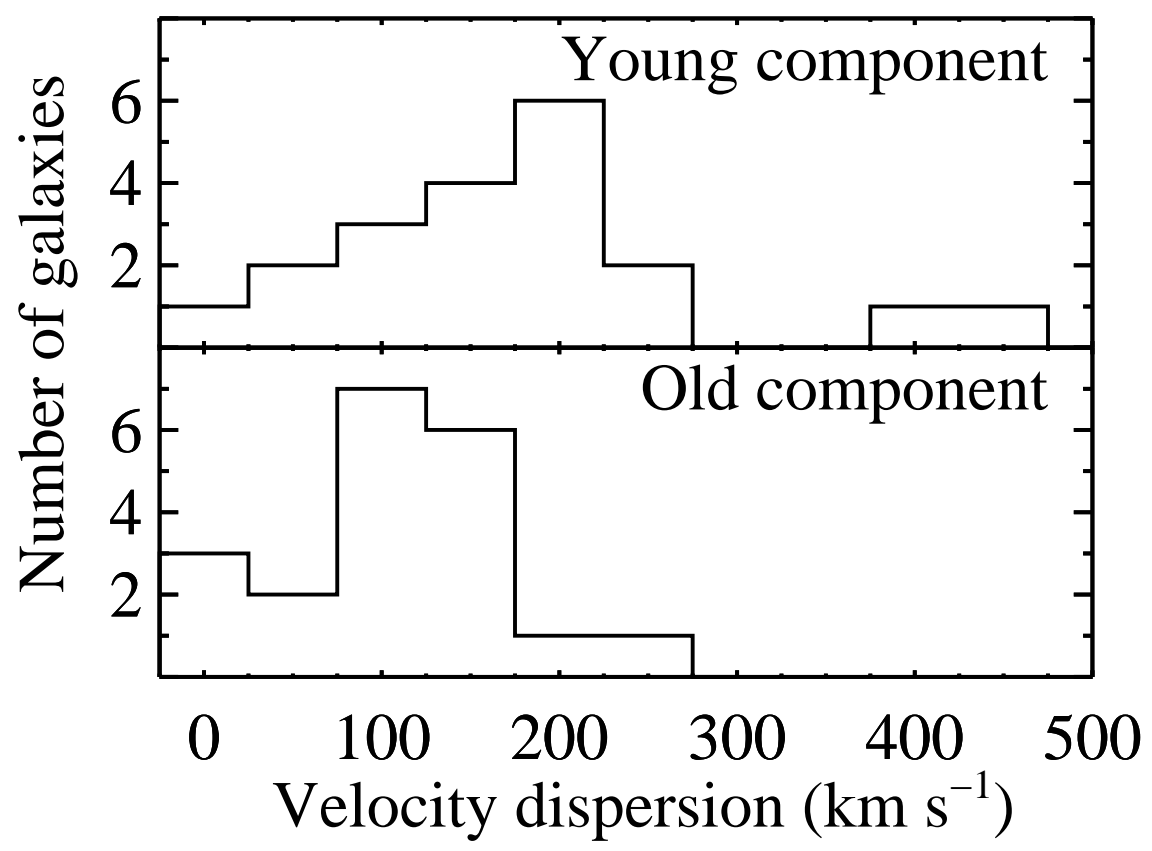

Fig. 14. - Histogram of velocity dispersion in the "total galaxy" aperture for each stellar population. On average, the younger population has a larger dispersion (see text for details and caveats). 


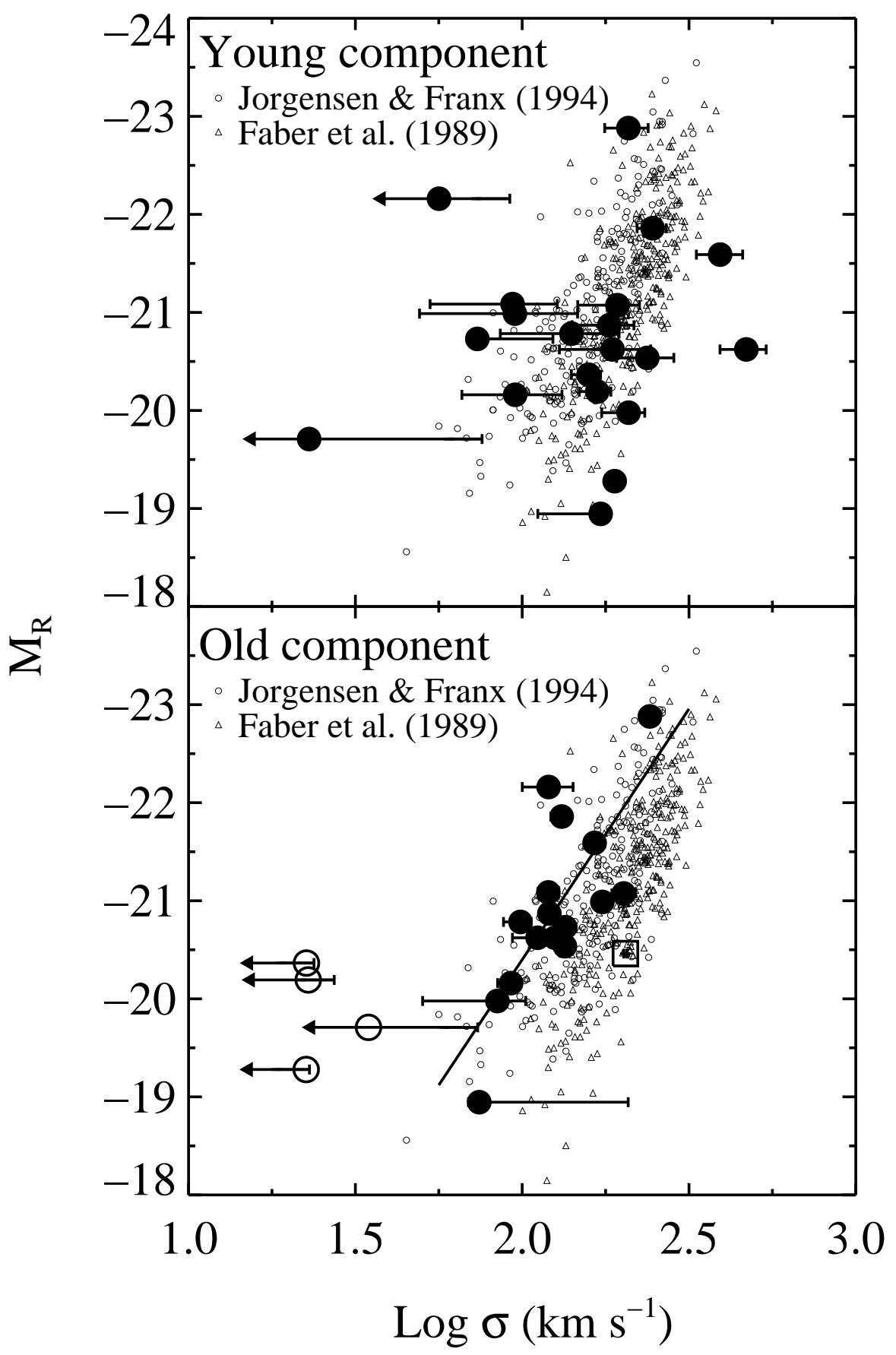

Fig. 15.- The luminosity-velocity dispersion relation for the E+A galaxies (open and filled large circles). The solid line represents a least-squares fit, weighting all points equally, to the filled points in the bottom panel; four outliers (open circles) are excluded from the fit. The large open square denotes EA 20, which has no young component and is not truly an E + A galaxy $(\S 3)$. Also plotted are normal elliptical galaxies from two studies (small circles and triangles; Faber et al. (1989), Jørgensen \& Franx (1994), respectively). 
0.6, with a significance of 99.8\%). We plot the best-fitting linear relation for the E+As for comparison, weighting all points equally, but excluding the four galaxies with only upper limits on $\sigma$. The scatter about this line, $0.6 \mathrm{mag}$, is the same as that around the relation for normal ellipticals (we measure a dispersion of 0.64 mag about the mean relation for the combined comparison sample). The faintest galaxies $\left(M_{R} \gtrsim-20\right)$, at least those with only upper limits on $\sigma$, are the outliers from this relation. The four outliers are drawn from the seven $\mathrm{E}+\mathrm{As}$ with the largest values of $\mathrm{f}_{A}$ (including EA 1 and the two other galaxies with the largest values of $\mathrm{f}_{A}$ ), suggesting that these galaxies may harbor more recent, unrelaxed mergers (like EA 1). On average, excluding these outliers, the E+As lie slightly to the lowdispersion (or high-luminosity) side of the elliptical galaxy relation, presumably at least in part because the young stellar populations elevate the luminosities. The offset in magnitude from Figure 15 is such that the $\mathrm{E}+\mathrm{A}$ galaxies are, on average, $\sim 0.6$ magnitudes brighter than their elliptical counterparts. Models (Poggianti et al. 1999; Kelson et al. 2000) show that over the next few Gyr the young stars in the E+A galaxies should experience dimming of approximately 0.5 to $1 \mathrm{mag}$, after which these galaxies would lie directly on the locus of elliptical galaxies.

To examine the outliers in relation to the rest of the $\mathrm{E}+\mathrm{A}$ sample, we plot the ratio of rotational velocity to velocity dispersion $(\mathrm{v} / \sigma)$ versus magnitude (Figure 16), adopting $\left\langle\mathrm{v}_{\text {rot }}\right\rangle$ of the old population for $\mathrm{v}$. As described in $\$ 3.2 .1$, the rotation velocities are uncorrected for inclination, so all $\mathrm{v} / \sigma$ points in Figure 16 are lower limits. Because we are concerned that spatial resolution may affect this measurement, we have divided the sample: the eight least-resolved galaxies (EA 1, 3, 5, 6, 10, 11, 15, and 21, as discussed in Section 3.1) are denoted by open circles, and the remaining galaxies are represented by filled circles. The poorly-resolved galaxies span a large range in $\mathrm{v} / \sigma$, so we conclude that low spatial resolution, which might cause us to underestimate the rotational velocities and overestimate velocity dispersions, is not grossly biasing our results toward low $\mathrm{v} / \sigma$. E+As fainter than $\mathrm{M}_{\mathrm{R}} \sim-20$ are limited to high $\mathrm{v} / \sigma$, and are thus rotationally supported. Previous work (Davies et al. 1983; Bender \& Nieto 1990) has similarly found that for $\mathrm{M}_{\mathrm{B}} \gtrsim-20$, normal ellipticals are flattened by rotation rather than by anisotropic velocity dispersions. The remainder of the sample (with $\mathrm{M}_{\mathrm{R}}<-20$ ) have an average $\mathrm{v} / \sigma$ of 0.32 .

From the analysis of velocity dispersions we reach three conclusions. First, the young populations have on average a higher velocity dispersion than the old populations. Three factors which may contribute to this result are: (a) the spatial segregation of the two populations (the young stars are more centrally concentrated and therefore lie deeper in the potential and have a higher velocity dispersion), (b) a transient, non-relaxed young component (the large observed $\sigma$ does not yet reflect an equilibrium value), and (c) a relaxed young component with kinematics distinct from those of the older component (as would arise if the 


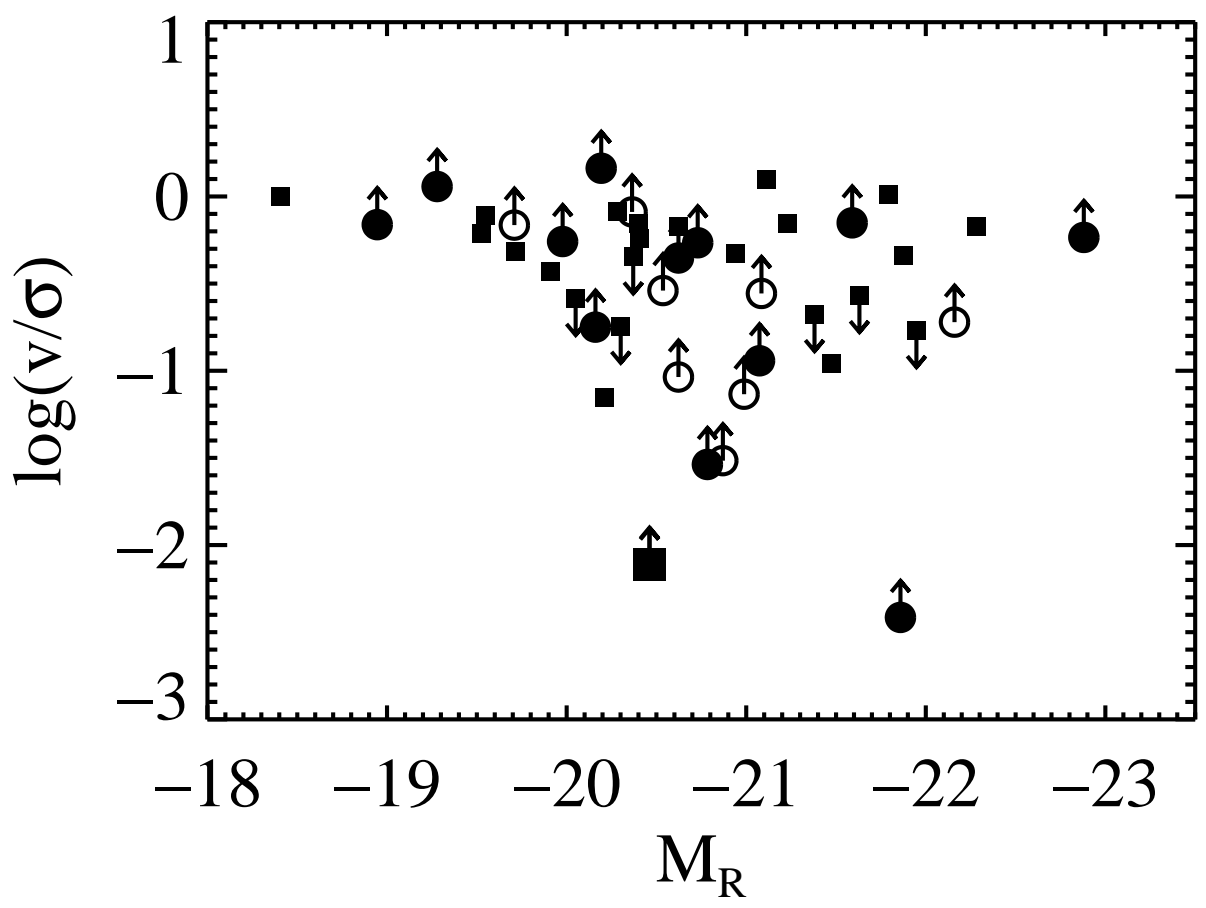

Fig. 16. - v/ $\sigma$ plotted against R-band absolute magnitude. Large circles (both open and filled) represent our E+A sample. Small squares represent elliptical galaxies from Davies et al. (1983) and Bender \& Nieto (1990) for which R-band magnitudes are available from the NASA/IPAC Extragalactic Database (NED). Rotational velocities in our sample are not corrected for inclination, so our points are marked as lower limits. Unfilled circles are galaxies that are only marginally resolved (see Section 3.1), but we find no systematic differences in $\mathrm{v} / \sigma$ between these and the other galaxies in our sample. 
A stars trace the formation of a hotter stellar component, such as a bulge). We cannot yet differentiate among these alternatives.

Second, the old component of the E+A galaxies conforms to the Faber-Jackson relation, although the E+As are somewhat brighter $(\sim 0.6 \mathrm{mag})$ than typical ellipticals. This result, combined with our finding that there is little evidence for rotation in most of the sample, indicates that the galaxies are in general, but not exclusively, supported by pressure rather than rotation.

Third, we find that the $\mathrm{E}+\mathrm{A}$ phase is experienced by a wide variety of galaxies. The velocity dispersions of $\mathrm{E}+\mathrm{As}$ in our sample range from $\lesssim 30 \mathrm{~km} \mathrm{~s}^{-1}$ to over $200 \mathrm{~km} \mathrm{~s}^{-1}$. Some E+As show significant rotation, but most lie on the Faber-Jackson relation, and rotational support becomes increasingly important for fainter E+As - just as it does for ellipticals.

\section{Discussion}

$\mathrm{E}+\mathrm{A}$ galaxies may represent a short lived phase in the evolution of many galaxies. Z96 estimated that $\sim 0.2 \%$ of all nearby galaxies are currently observed in an extreme $\mathrm{E}+\mathrm{A}$ phase. For an estimated lifetime of the $\mathrm{E}+\mathrm{A}$ phase of $\sim 1 \mathrm{Gyr}$, their result suggests that $\gtrsim 3 \%$ of galaxies may have experienced such a phase in a Hubble time. This is a relatively small fraction of all galaxies, but if $\mathrm{E}+\mathrm{As}$ are associated primarily with elliptical galaxies and the field elliptical fraction is $10 \%$ today, the fraction of ellipticals that passed through an $\mathrm{E}+\mathrm{A}$ phase at some point could be as high as $30 \% .{ }^{8}$ This estimate is subject to many uncertainties, but it demonstrates the potential importance of the $\mathrm{E}+\mathrm{A}$ phase in galaxy evolution. In addition, the number of $\mathrm{E}+\mathrm{A}$-like systems over a Hubble time may actually be underestimated here, because of (1) the extremely conservative E+A criteria used by Z96, (2) the short lifetime of the extreme E+A phase (less than $1 \mathrm{Gyr}$ ), and (3) the larger fraction of E+As in the past (Dressler \& Gunn 1983; Belloni et al. 1995). As observers probe the properties of galaxies at earlier and earlier times, it becomes increasingly important that we understand local counterparts to the distant post-starburst galaxy population. E+As provide us with a rare opportunity to study galaxies in transition.

Our measurements of $\sigma, \mathrm{v} / \sigma$, and the distributions of young and old stars indicate that $\mathrm{E}+\mathrm{As}$ are not, as a class, disk galaxies that have had their star formation quenched

\footnotetext{
${ }^{8}$ This result is not in conflict with the observational constraints on the lack of young populations in early type galaxies (Silva \& Bothun 1998). Even if our estimate of $30 \%$ is correct, we would predict only one galaxy in the Silva \& Bothun sample to have a significant 1 to 3 Gyr old population, and they observe no galaxies with such populations contributing at greater than the 10 to $15 \%$ level.
} 
(Newberry, Boroson \& Kirshner 1990) or that have experienced a burst followed by a cessation of star formation as a result of interaction with the intracluster medium (Dressler \& Gunn 1983). However, one model does not fit all of our E+As. In particular, a few of our systems have significant $\left(>50 \mathrm{~km} \mathrm{~s}^{-1}\right.$ ) rotation velocities. Yet only two of these systems have $\mathrm{v} / \sigma(\sin i)>1$, and so only a small fraction of the sample is predominantly rotationally supported. This low fraction seems to be at odds with the high fraction of such systems among the published kinematic studies of E+As (Franx 1993; Caldwell et al. 1996). However, one of the three fast-rotating E+As in the Coma sample, NGC 4853, has detectable [OII] emission and similar galaxies have been excluded from the Z96 sample. The other two Coma cluster fast-rotators have $M_{R}>-20$, and so are comparable to the four low-luminosity, rotationally-supported $\mathrm{E}+$ As in our sample. Because of these circumstances, we conclude that our results are not in disagreement with previously published studies.

Increasingly, it is generally accepted that at least some fraction of $\mathrm{E}+\mathrm{A}$ galaxies are the result of a galaxy merger (e.g., Lavery \& Henry (1988); Schweizer (1996), Z96). There is an abundance of circumstantial evidence supporting this scenario, from the observations of tidal tails in several galaxies in this sample (Z96) to the kinematic observations we present that suggest that $\mathrm{E}+\mathrm{As}$ are a link between star forming galaxies (which are likely gas-rich disks) and quiescent, spheroidal galaxies that lie on the Faber-Jackson relation and have low $\mathrm{v} / \sigma$. In a merger model for the formation of $\mathrm{E}+\mathrm{As}$, our observation that the young stars are more concentrated toward the galaxy center than are the older stars indicates that the gas that formed the younger stars dissipated energy before the starburst. Such dissipation is required for the formation of elliptical galaxies from merging spirals, in order to explain how the cores of elliptical galaxies increased their phase space density beyond than of their spiral progenitors (Kormendy \& Sanders 1992). However, that dissipation does not drive all of the gas to the galaxy nucleus as seen in some simulations (cf. Mihos \& Hernquist (1996)), because the burst of star formation extends beyond the nucleus in at least seven of our E+As. The disagreement between observations and simulations hints at a problem with angular momentum transport or feedback in the simulations (Mihos \& Hernquist 1994). Alternatives have been suggested (e.g., Mihos, Bothun \& Richstone (1993)) in which the star formation is due to molecular cloud collisions (Noguchi 1988, 1991). Unfortunately, the results of such models are not yet directly comparable to our observations.

A complete model for the phenomena associated with this phase of galaxy evolution would relate the evolution of the morphological and dynamical properties of the galaxy to the evolution of its stellar populations. As such, it may at first appear difficult to effect the hypothesized dramatic structural changes on the short timescales implied by the stellar populations ( $\lesssim 1$ Gyr). However, violent relaxation (Lynden-Bell 1967) in mergers may reduce the timescale over which one expects global morphological changes to the order of 10 
crossing times $\left(\sim 10^{8}-10^{9}\right.$ yr; Borne (1984); Mihos (1995)). Gaseous dissipation can also significantly shorten this timescale (Mihos, Richstone \& Bothun 1992). Furthermore, the age constraint from stellar populations reflects the time since the last burst of star formation, which may come well after any initial interaction between progenitors (see Mihos, Bothun \& Richstone (1993) and Bekki (2001) for examples of simulations that predict multiple starbursts in merging systems). Recent models that take these effects into account (e.g., Mihos (1999)) predict merger dynamical timescales of $\sim 1-2$ Gyr. Therefore, although the synchronization of the dynamical and stellar timescales will continue to be a challenge for detailed modeling, we conclude that the two are fully reconcilable.

\section{Conclusions}

We obtain and analyze long-slit spectra to study the spatial distribution and kinematic properties of the old and young stellar populations of a local sample of E+A galaxies (Z96). We summarize our results from the study of these $20 \mathrm{E}+\mathrm{A}$ galaxies. ${ }^{9}$ We have four principal findings:

1. The star formation responsible for the young stellar populations in E+A galaxies is more centrally concentrated than the older stellar populations in most E+A galaxies in our sample, but is resolved beyond the nuclear region in seven cases.

2. The $\mathrm{E}+\mathrm{A}$ galaxies in our sample are principally pressure-supported. We find that only six of our $20 \mathrm{E}+\mathrm{As}$ are rotating with $\left\langle\mathrm{v}_{\text {rot }}\right\rangle \sin i \gtrsim 50 \mathrm{~km} \mathrm{~s}^{-1}$ in either the young or old populations. Though we are only able to measure the velocities at radii of $1-3 \mathrm{kpc}$, this is significantly less rotation than is typically observed at the same radius in normal spiral galaxies. We also find that the old stellar populations of $\mathrm{E}+\mathrm{A}$ galaxies conform well to the Faber-Jackson relation. This result argues against the model in which E+As, as a class, are normal spiral galaxies in which star formation has recently been extinguished. On average, the E+As are slightly ( $\sim 0.6 \mathrm{mag})$ brighter than a sample of typical elliptical galaxies for the same velocity dispersions. As the young populations in the E+As age and fade, this luminosity difference will disappear. The degree to which the E+As are already consistent with the Faber-Jackson relation demonstrates that $\mathrm{E}+\mathrm{A}$ galaxies relax quickly $(\lesssim 1 \mathrm{Gyr}$ ) to the locus of points occupied by typical ellipticals, which is consistent with the predictions of merger models.

\footnotetext{
${ }^{9}$ One of the original 21 galaxies, EA 20, was apparently misclassified due to low $\mathrm{S} / \mathrm{N}$ in the original LCRS spectrum.
} 
3. We find that the $\mathrm{E}+\mathrm{A}$ phase of galaxy evolution does not only produce bright spheroids, but galaxies that span the full range of the Faber-Jackson relation. Our sample includes a population of $\mathrm{E}+\mathrm{As}$ that resembles the low-luminosity class of ellipticals that are flattened by rotation (Davies et al. 1983; Bender \& Nieto 1990) and produced in high mass-ratio merger simulations (Cretton et al. 2001). Given the number of E+As and the duration of the $\mathrm{E}+\mathrm{A}$ phase, a simple estimate shows that one-third of field ellipticals could have passed through this phase at some point. This estimate, though it is not expected to predict the actual number of ellipticals that were once E+As, demonstrates the potential importance of this phase. Because E+As populate the entire Faber-Jackson relation, we conclude that the E+A phase may be one through which many spheroid-dominated galaxies pass.

4. Our kinematic observations are strong evidence that luminous $\left(\mathrm{M}_{\mathrm{R}}<-20\right) \mathrm{E}+\mathrm{A}$ galaxies are an evolutionary link between gas-rich, star-forming galaxies and gas-poor, quiescent, pressure-supported galaxies. Other authors have highlighted the spectral evidence for significant recent star formation in E+As (which demonstrates that these galaxies recently had substantial gas reservoirs) and against ongoing star formation (Couch \& Sharples 1987; Newberry, Boroson \& Kirshner 1990; Schweizer 1996; Chang et al. 2001). This study demonstrates that faded E+A galaxies will resemble early-type galaxies kinematically, as well as spectroscopically. The presence of tidal features in many (Z96) suggests disk-dominated progenitors. We conclude that at least most $\mathrm{E}+\mathrm{A}$ galaxies in our sample are in the midst of a morphological transition from late-type (star-forming, gas-rich, rotationally-supported, and disk-dominated) to early-type (quiescent, gas poor, pressure-supported, spheroid-dominated).

The authors thank Chris Mihos for his comments on interaction scenarios, John Mulchaey for his observing expertise, and Greg Bothun for his helpful comments on the manuscript. We also thank Luc Simard for introducing us to the NUKECR algorithm which we used to effectively remove cosmic rays. This research has made use of the NASA/IPAC Extragalactic Database (NED) which is operated by the Jet Propulsion Laboratory, California Institute of Technology, under contract with the National Aeronautics and Space Administration. The data described herein were taken at Las Campanas observatory, a facility of Carnegie Observatories. AIZ acknowledges financial support from NASA grant HF-01087.01-96A. DZ acknowledges financial support from the David and Lucile Packard Foundation, the Alfred P. Sloan Foundation, a NASA LTSA grant (NAG 5-3501), and an NSF CAREER grant (AST-9733111). 


\section{REFERENCES}

Barnes, J. E. \& Hernquist, L. E. 1991, ApJ, 370, L65

Bekki, K. 2001, ApJ, 546, 189

Belloni, P., Bruzual, A. G., Thimm, G. J.,\& Roser, H.-J., å, 297, 61

Bender, R., \& Nieto, J.-L. 1990, A\&A, 239, 97

Blanton, et al., 2001, AJ, submitted

Borne, K. D. 1984, ApJ, 287, 503

Bothun, G. \& Dressler, A. 1986, AJ, 301, 57

Bressan, A., Fagotto, F., Bertelli, G., \& Chiosi, C. 1993, A\&AS, 100, 647

Bruzual, A. G., \& Charlot, S. 1993, ApJ, 405, 538

Caldwell, N., Rose, J. A., Franx, M., \& Leonardi, A. J. 1996, ApJ, 111, 78

Chang, T.-C., van Gorkom, J. H., Zabludoff, A. I., Zaritsky, D., \& Mihos, J. C., 2001, AJ submitted

Couch, W. J. \& Sharples, R. M. 1987, MNRAS229, 423

Cretton, N., Naab, T., Rix, H.-W., \& Brukert, A, 2001, ApJ, submitted

Davies, R. L. 1981, MNRAS194, 879

Davies, R. L., Efstathiou, G., Fall, S. M., Illingworth, G., \& Schechter, P. L. 1983, ApJ, 266, 41

Dressler, A., Gunn, J. E. 1983, ApJ, 270, 7

Faber, S. M., \& Jackson, R. E. 1976, ApJ, 204, 668

Faber, S., Wegner, G., Burstein, D., Davies, R., Dressler, A., Lynden-Bell, D., \& Terlevich, R. 1989, ApJS, 69, 763

Franx, M. 1993, ApJ, 407, L5

Gebhardt, K., Bender, R., et al. 2000, ApJ, 539, L13

Gebhardt, K., Faber, S., et al. 2001, ApJ, submitted. 
Héraudeau, Ph., Simien, F., Maubon, G., \& Prugniel, Ph. 1999, A\&AS, 136, 509

Jog, C. J., \& Solomon, P. M. 1992, ApJ, 387, 152

Jørgensen, I., \& Franx, M. 1994, ApJ, 433, 553

Kelson, D. D., Illingworth, G. D., van Dokkum, P. G., Franx, M. 2000, ApJ, 531, 184

Kormendy, j. \& Sanders, D. B. 1992, ApJ, 390, L53

Lavery, R., \& Henry, P. 1988, ApJ, 330, 596

Leitherer, C., et al. . 1996, PASP, 108, 996

Lin, H., Kirshner, R. P., Shectman, S. A., Landy, S. D., Oemler, A., Tucker, D. L., Schechter, P.L. 1996, ApJ, 464, 60

Liu, C. T. \& Kennicutt, R. C. 1995, ApJ, 450, 547

Lynden-Bell, D. 1967, MNRAS, 136, 101

Mihos, J. C. 1995, ApJ, 438, L75

Mihos, J. C. 1999, in ASP Conf. Ser. 197, Galaxy Dynamics: from the Early Universe to the Present, eds. F. Combes, G. A. Mamon, \& V. Charmandaris (San Francisco: ASP), 275

Mihos, J. S. \& Bothun, G. D. 1998, ApJ, 500, 619

Mihos, J. C., Bothun, G. D., Richstone, D. O. 1993, ApJ, 418, 82

Mihos, J. C., \& Hernquist, L. 1994, ApJ, 437, L47

Mihos, J. C., \& Hernquist, L. 1996, ApJ, 464, 641

Mihos, J. C., Richstone, D. O., \& Bothun, G. D. 1992, ApJ, 400, 153

Moore, B., Lake, G., \& Katz, N. 1998, ApJ, 495, 139

Newberry, M.V., Boroson, T.A., Kirshner, R.P. 1990, ApJ, 350, 585

Noguchi, M. 1988, A\&A, 203, 259

Noguchi, M. 1991, MNRAS, 251, 360

Oegerle, W. R., Hill, J. M., \& Hoessel, J. G. 1991, ApJ, 381, L9 
Poggianti, B. M., Smail, I., Dressler, A., Couch, W. J., Barger, A. J., Butcher, H., Ellis, R. S., \& Oemler, A. 1999, ApJ, 518, 576

Rieke, G.H., Cutri, R.M., Black, J.H., Kailey, W.F., McAlary, C.W., Lebofsky, M.J., \& Elston, R. 1985, ApJ, 290, 116

Schweizer, F. 1982, ApJ252, 455

Schweizer, F. 1996, AJ, 111, 109

Shectman, S. A., Landy, S. D., Oemler, A. A., Tucker, D., Lin, H., Kirshner, R. P., \& Schechter, P. L. 1996, ApJ, 470, 172

Shectman, S. A., Schechter, P. L., Oemler, A. A., Tucker, D., Kirshner, R. P., \& Lin, H. 1992, in Clusters and Superclusters of Galaxies, ed. A. C. Fabian (Dordrecht:Kluwer), 351

Silva, D.R., \& Bothun, G.D. 1998, AJ, 116, 85

Smail, I., Morrison, G., Gray, M. E., Owen, F. N., Ivison, R. J., Kneib, J.-P., \& Ellis, R. S. 1999, ApJ, 525, 609

Toomre, A., in The Evolution of Galaxies and Stellar Populations, edited by B.M. Tinsley and R.B. Larson (New Haven: Yale Univ.), p. 401

Toomre, A., \& Toomre, J. 1972, ApJ, 178, 623

van der Marel, R., \& Franx, M. 1993, ApJ, 407, 525

Windhorst, R. A., Franklin, B. E., \& Neuschaefer, L. W. 1994, PASP, 106, 798

Wright, G. S., Joseph, R. D., Robertson, N. A., James, P. A., \& Meikle, W. P. S. 1988, MNRAS, 233, 1

Zabludoff, A. I., Zaritsky, D., Lin, H., Tucker, D., Hashimoto, Y., Shectman, S.A., Oemler, A. A., \& Kirshner, R. P. 1996, ApJ, 466, 104 